Research Article

\title{
Combining of the H/A/Alpha and Freeman-Durden Polarization Decomposition Methods for Soil Moisture Retrieval from Full-Polarization Radarsat-2 Data
}

\author{
Qiuxia Xie, ${ }^{1,2,3}$ Qingyan Meng $\mathbb{D}^{1,3}$ Linlin Zhang $\mathbb{D}^{1,2,3}$ Chunmei Wang, ${ }^{1,3}$ Qiao Wang, \\ and Shaohua Zhao ${ }^{4}$ \\ ${ }^{1}$ Institute of Remote Sensing and Digital Earth, Chinese Academy of Sciences, Beijing 100101, China \\ ${ }^{2}$ University of Chinese Academy of Sciences, Beijing 100101, China \\ ${ }^{3}$ Sanya Institute of Remote Sensing, Sanya 572029, China \\ ${ }^{4}$ Satellite Environment Center, Ministry of Environmental Protection, Beijing 100094, China
}

Correspondence should be addressed to Qingyan Meng; mengqy@radi.ac.cn

Received 5 February 2018; Revised 26 June 2018; Accepted 5 July 2018; Published 19 August 2018

Academic Editor: Stefania Bonafoni

Copyright (c) 2018 Qiuxia Xie et al. This is an open access article distributed under the Creative Commons Attribution License, which permits unrestricted use, distribution, and reproduction in any medium, provided the original work is properly cited.

Soil moisture (SM) plays important roles in surface energy conversion, crop growth, environmental protection, and drought monitoring. As crops grow, the associated vegetation seriously affects the ability of satellites to retrieve SM data. Here, we collected such data at different growth stages of maize using Bragg and X-Bragg scattering models based on the Freeman-Durden polarization decomposition method. We used the H/A/Alpha polarization decomposition approach to extract accurate threshold values of decomposed scattering components. The results showed that the $\mathrm{H}$ and Alpha values of bare soil areas were lower and those of vegetated areas were higher. The threshold values of the three scattering components were $0.2-0.4 \mathrm{H}$ and $7-24^{\circ}$ Alpha for the surface scattering component, $0.6-0.9 \mathrm{H}$ and $22-50^{\circ}$ Alpha for the volume scattering component, and other values for the dihedral scattering component. The SM data retrieved (using the X-Bragg model) on June 27, 2014, were better than those retrieved at other maize growth stages and were thus associated with the minimum root-mean-square error value $(0.028)$. The satellite-evaluated SM contents were in broad agreement with data measured in situ. Our algorithm thus improves the accuracy of SM data retrieval from synthetic-aperture radar (SAR) images.

\section{Introduction}

Soil moisture content (SMC) is a key in study of agricultural production, environmental protection, and surface energy conversion, such as drought monitoring and dust storm monitoring [1-3]. SMC can be retrieved by evaluating the backscattering coefficients associated with microwave remote sensing data. A variety of theoretical, empirical, and semiempirical SM retrieval models have been developed, including the classical IEM, AIEM, Oh, Dubois, and Shi models [4]. However, vegetation adversely affects the reliability of SMC retrieval [5]. It is difficult to distinguish the various scattering components of vegetation-covered surfaces [6]. The radar was frequently used in detecting SMC; however, the signals are attenuated by vegetation. Therefore, the sensitivity of the radar signal detecting SMC is reduced by vegetation. This is a major problem for soil moisture retrieval from radar data [7]. Water cloud model is widely used in evaluating low vegetation area. It is the most common retrieval method used [5]. This model features only two scattering mechanisms: surface scattering from bare soil and volume scattering from vegetation [6]. As the crop grows, the scattering mechanisms change. Surface scattering changes with the growth of the crop. Therefore, we need to study the scattering at different growth stages. It is difficult to retrieve the SMC using only water cloud model at different growth stage of maize [8].

C-band Radarsat-2 has the characteristics of full polarization with higher resolution $(8 \mathrm{~m})$. C-band Radarsat-2 could provide more information in SMC retrieval by 
full-polarization characteristics $(\mathrm{HH}, \mathrm{VV}, \mathrm{HV}$, and $\mathrm{VH})$ [9]. The data can be decomposed into various scattering mechanisms (two-, three-, and four-component mechanisms) [10]. Currently, the principal decomposition methods used are the Freeman-Durden, Yamaguchi, Vanzly, H/A/Alpha, and Arii algorithms [11], particularly the Freeman-Durden method [12]. The SAR data are divided into a surface scattering component associated with bare soil, a volume scattering component associated with vegetation, and a dihedral scattering component associated with both bare soil and vegetation [13]. The advantages of the method include simple direct separation of the components and elimination of vegetation scattering signals. The surface scattering component pertains to bare soil only. Theoretically, SMC retrieval is more accurate when the surface scattering component is used. Therefore, it is essential to maximize the surface scattering component when building an SMC retrieval model. However, the decomposition of the data is a challenge. The $H / A$ /Alpha polarization decomposition method divides the full-polarization SAR data into a scattering entropy $(H)$, an antientropy $(A)$, and an alpha angle (Alpha) [11]. Using this full-polarization model, Cloude and Pottier divided the feature spaces of $H$ and Alpha into eight effective areas [14], each of which corresponded to a specific scattering mechanism. Usually, the cutoff value of $H$ is 0.5 and that of Alpha is $45^{\circ}$, between surface and vegetation scattering [15]. Based on the feature spaces of $H$ and Alpha, surface, volume, and dihedral scattering components can be accurately extracted [16].

In this study, we used the H/A/Alpha and FreemanDurden polarization decomposition methods to retrieve SMC at different growth stages of maize (seeding, jointing, heading, and flowering). We incorporated the H/A/Alpha method to increase the accuracies of the three scattering components (surface, volume, and dihedral). Backscattering and threshold analyses of typical objects were evaluated by calculating backscattering coefficients and the feature spaces of $H$ and Alpha. Using both the Bragg and X-Bragg scattering models, SMC was retrieved from the surface scattering data obtained at different maize growth stages. The advantages of this approach are that the $H / A$ /Alpha method yields quantitative SMC measures using three highly accurate scattering components to retrieve SMC data at different maize growth stages. Thus, SMC retrieval was possible employing a polarization decomposition technique.

\section{Study Site and Dataset}

2.1. Study Site. Our study site was near Hengshui City, Hebei Province, in the northern plain of China $\left(38^{\circ} 3^{\prime} 00^{\prime \prime} \mathrm{N}\right.$, $115^{\circ} 27^{\prime} 54^{\prime \prime} \mathrm{E}$ ) (Figure 1). The plain is also termed the Huang Huai Hai plain and is one of the three major plains of China. Our study site was a typical agricultural region. The major summer crops are maize (ca. $80 \%$ ), cotton, and peanuts. This study site was approximately $25 \times 25 \mathrm{~km}$ in area, and it is dominated by a loamy soil $(37.9 \%$ sand, $44.6 \%$ silt, and $17.5 \%$ clay (all w/w)) [17]. The terrain is almost flat, the soil is homogeneous, and maize is the preferred crop. The climate is continental, characterized by high temperature and severe drought in summer. Most crop growth occurs in summer, at which time the SMC is critical [17].

The maize growing period runs from June to September. Therefore, we performed four field experiments on June 24, July 24, August 14, and September 4, when maize was at the sowing, jointing, heading, and flowering stages, respectively. At the sowing stage, vegetation is lacking (the soil is bare). However, small areas were covered with residual wheat stalks, grass, fruit trees, or early-planted maize. Therefore, use of the bare soil moisture content retrieval method alone was inappropriate; it was necessary to distinguish between various land cover types and adopt our methods accordingly to ensure data accuracy. At the jointing stage, the leaf area index (LAI) of maize is about 1.5 , the mean maize height $(\mathrm{MH})$ about $50 \mathrm{~cm}$, and the mean leaf age index (LAGI) about 30\%; the wheat stalks disappear gradually. At the heading stage, the LAI is about 3.0, the MH about $150 \mathrm{~cm}$, and the LAGI about $70 \%$. At the flowering stage, the LAI is about 3.5, the $\mathrm{MH}$ about $200 \mathrm{~cm}$, and the LAGI about $80 \%$ [17].

2.2. Dataset. In this study, data from Radarsat-2 and GF-1 (the first high-resolution satellite) were used to retrieve SMC based on a combination of the H/A/Alpha and FreemanDurden polarization decomposition methods. The C-band full-polarization synthetic-aperture radar (SAR) data of the Radarsat-2 high-resolution radar satellite were acquired by the Canadian Space Agency and the MDA Corporation in 2007 [18]. Of the four Radarsat-2 polarization products, the single-look complex (SLC) product is a slanted-range dataset affording optimal resolution, amplitude, and phase information for all beam patterns. Four SLC product scenarios were selected with 8-meter spatial resolution, time resolution of 24 days, central incidence angle of $39^{\circ}$, and swath width of $25 \mathrm{~km}$ in the quad-polarization beam mode (Q19) [19]. The GF-1 satellite is a multispectral satellite launched by China in 2013 and affords 8-meter spatial resolution and a time resolution of 4 days $[20,21]$. Various ground objects can be distinguished using the GF-1 multispectral data [22].

It was necessary to preprocess both datasets to allow SMC retrieval via polarization decomposition methods [23]. We used ENVI 5.3 software and the NEXT ESA SAR Toolbox to preprocess Radarsat-2 SLC data in terms of multilooking, radiometric correction, geocoding, filtering, and processing. Filtering was used to eliminate speckle noise. During processing of multilooking, the spatial resolutions of SLC data are reduced, but the radiation resolution (which imparts intensity information) improves. GF-1 preprocessing includes radiometric calibration, atmospheric and geometric corrections, and image cropping [23]. After such preprocessing, GF-1 distinguished different groundcover types well. We used the GF-1 data as auxiliary in terms of SMC retrieval. The data reveal the spatial characteristics and differences among ground objects such as bare soil, urban/rural areas, orchards, grassland, and water bodies.

The four filed experimental days were chosen to coincide with the passage of Radarsat-2 (Table 1). We measured soil and vegetation parameters at 23 study sites (Figure 1). All of 23 study sites were the maize fields. For the convenience of 


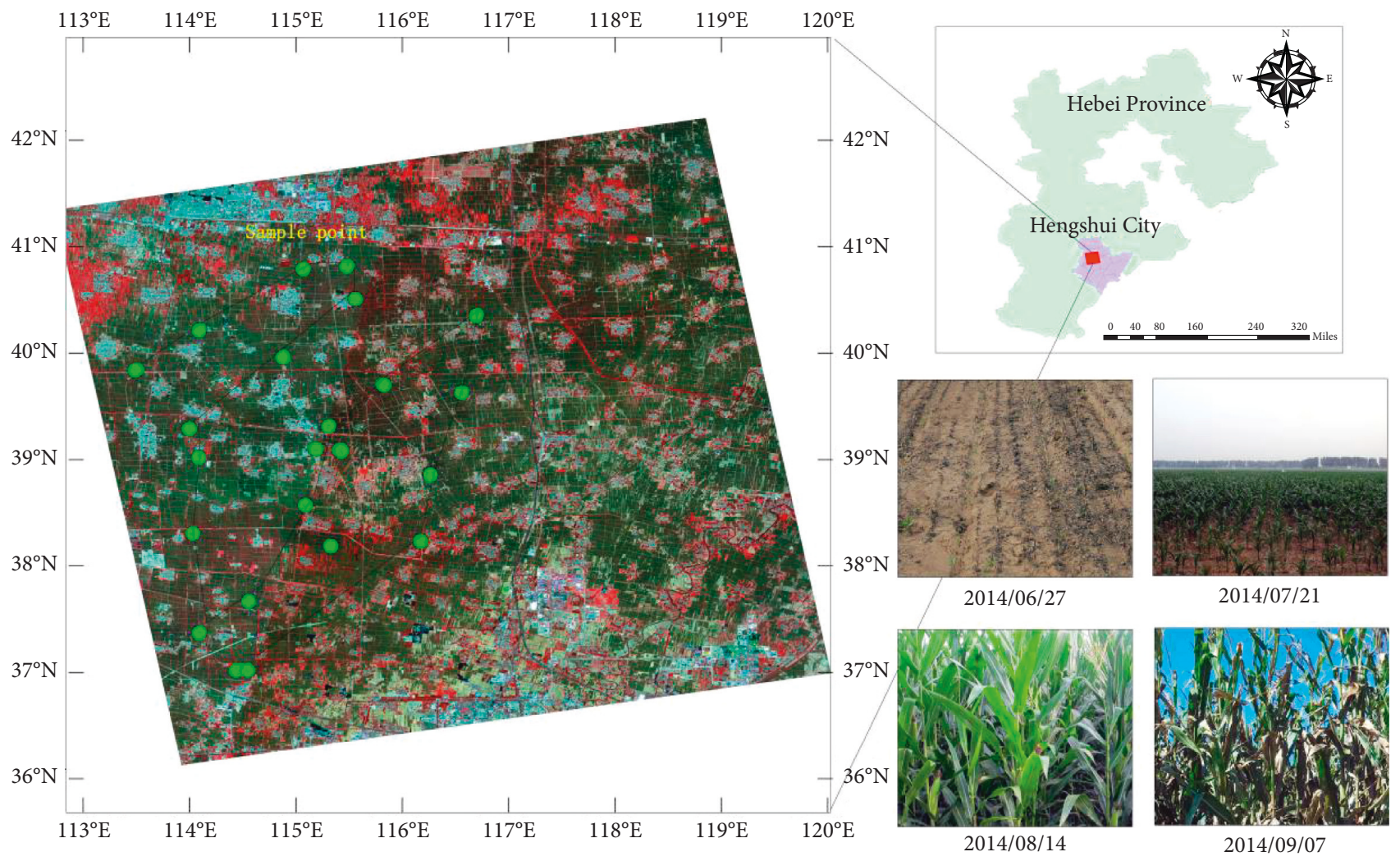

FIgURE 1: Location of the study and the four experiments.

TABLE 1: Radarsat-2 and in situ measurement.

\begin{tabular}{|c|c|c|c|c|c|c|c|c|c|c|}
\hline $\begin{array}{l}\text { Date } \\
\text { (yyyy/mm/dd) }\end{array}$ & $\begin{array}{l}\text { Time } \\
\text { UTM }\end{array}$ & Dominant obje & SPN & $\begin{array}{c}\mathrm{SMC} \\
\left(\mathrm{g} / \mathrm{cm}^{3}\right)\end{array}$ & $\begin{array}{c}\text { EWT } \\
\left(\mathrm{kg} / \mathrm{m}^{2}\right)\end{array}$ & $\varepsilon_{r}$ & $H(\mathrm{~m})$ & $\rho_{\text {soil }}$ & $\begin{array}{c}\text { RMS_h } \\
(\mathrm{cm})\end{array}$ & LAI \\
\hline $2014 / 06 / 27$ & $10: 17: 30$ & Bar & 6 & $5.62-48.1$ & 0 & 2.3 & . & 1.578 & $0.4-3.4$ & 0 \\
\hline $2014 / 07 / 21$ & $0: 17: 30$ & 3are soil/ma & 6 & $6.4-$ & $0.05-2$ & $1.3-$ & & 1.27 & & 1.37 \\
\hline 201 & $10: 17: 30$ & Maize a & 6 & $9.1-$ & $0.48-$ & $3.7-$ & 2 & 1. & 3.0 & 2.89 \\
\hline $2014 / 09 / 07$ & $10: 17: 30$ & Maize area/orchard & 60 & $8.6-39.3$ & $1.53-3.90$ & $3.4-17.8$ & 2.58 & 1.33 & $0.4-2.2$ & 3.35 \\
\hline
\end{tabular}

SPN: sample point number; $\varepsilon_{r}$ : soil dielectric constant; $\rho_{\text {soil }}$ : mean soil bulk density; EWT: vegetation canopy water content; $H$ : mean maize height.

the experiment, the route of 23 study sites was set up along the road. We assessed SMC, the soil dielectric constant, surface roughness, vegetation water content (EWT), the LAI, and maize height. All parameters were measured three times, and the means were calculated. Half of the data were used to build SMC retrieval model, and the other half were used to verify the models.

The SMC is the most important field parameter. The $\mathrm{C}$-band penetrates the surface to only about $5 \mathrm{~cm}$. Therefore, we measured SMC at that depth using an aluminum box and a $200 \mathrm{~cm}$ diameter cutting ring. Then, the soil samples were weighed, brought to the laboratory, and dried at $105^{\circ} \mathrm{C}$ for about $24 \mathrm{~h}$. The SMC was calculated as the difference between the dry and wet weights. The soil dielectric constant was measured in the same laboratory samples with the aid of an E5071C vector network analyzer (Keysight Technologies, Inc., Santa Rosa, CA, USA). Roughness was estimated using a plate of $1 \mathrm{~m}$ in length bearing 101 pins; we measured the root-mean-square heights (RMS_h) and the correlation lengths $(L)$ in two directions ( $\mathrm{N}$ to $\mathrm{S}$ and $\mathrm{E}$ to $\mathrm{W}$ ) and calculated the means. We also measured vegetation parameters at different growth stages. LAI was measured five times with the aid of an LAI-2200C plant canopy analyzer (LI-COR Biosciences, Lincoln, NE, USA), and the data were averaged. Vegetation water content was calculated as the difference between the wet and dry weights. Fresh maize (including the green portions) was dried at $105^{\circ} \mathrm{C}$ for $40 \mathrm{~min}$ and then at $85^{\circ} \mathrm{C}$ for $48 \mathrm{~h}$.

\section{Methodology}

Vegetation greatly affects SMC retrieval from SAR data. As ground scattering signals are attenuated by vegetation, ground data are difficult to obtain, compromising SMC retrieval. To eliminate the effects of vegetation, we combined two polarization decomposition techniques (H/A/Alpha and Freeman-Durden) [24]. The specific step is shown in Figure 2 for soil moisture retrieval. 


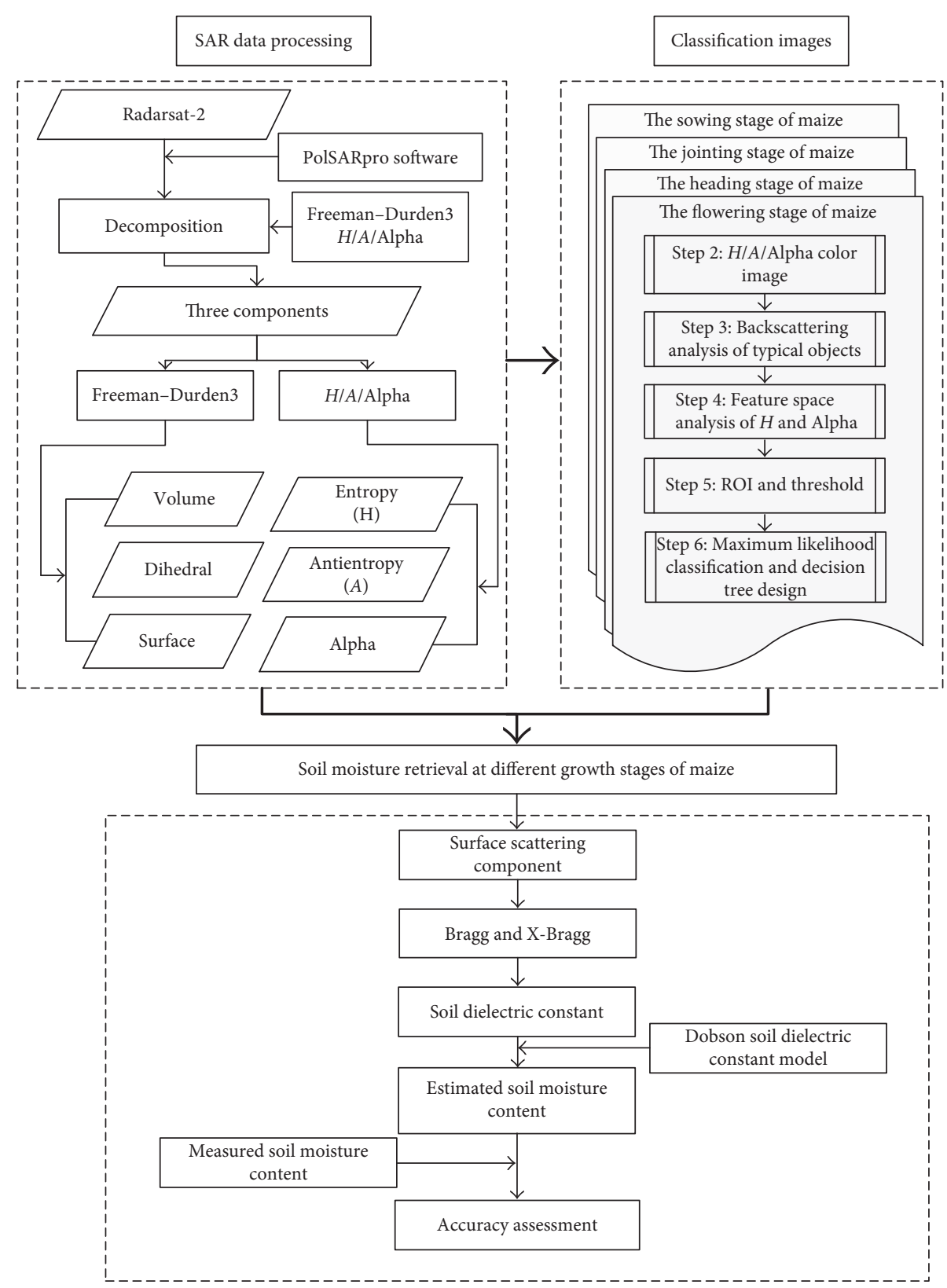

FIGURE 2: Flowchart of soil moisture content retrieval.

3.1. The H/A/Alpha Method. Based on the electromagnetic theory, the different polarization types of SAR data can be obtained, such as HH, VV, and HV. Different polarization SAR data reflected the different characteristics between the object and electromagnetic wave. When ground objects and radar signals interact, backscattering of objects is affected by their characteristics and the type of polarization used. Therefore, ground objects appear different depending on the chosen polarization, allowing the various objects to be distinguished and classified.

H/A/Alpha is a polarization decomposition method based on eigen decomposition of polarized SAR data [18]. The biggest advantage of eigen decomposition is that it is not limited by the specific scattering mechanism employed; the eigenvalues do not change with transformation of the antenna coordinate system [25]. Therefore, the method is associated with invariance in the basis, including rotation invariance, rendering the data more reliable.

Backscattering information obtained by radar can be expressed as a scattering matrix, as follows:

$$
\mathbf{S}=\left[\begin{array}{ll}
S_{h h} & S_{h v} \\
S_{v h} & S_{v v}
\end{array}\right]
$$

After Pauli decomposition of this matrix, the $\mathbf{K}$ vector is given by

$$
\mathbf{K}=\frac{1}{\sqrt{2}}\left[\begin{array}{lll}
S_{h h}+S_{v v} & S_{h h}-S_{v v} & 2 S_{h v}
\end{array}\right] .
$$

As the Pauli basis is orthogonal, the three components of the $\mathbf{K}$ vector are interrelated. Therefore, SAR data are usually 
decomposed based on the amplitudes or powers of these three items. The two-order matrix of the $\mathbf{K}$ vector is regarded as a $\left[\mathbf{T}_{3}\right]$ matrix and also as a $\mathbf{U}$ matrix [26]. The $\mathbf{U}$ matrix is given by

$$
\mathbf{T}_{3}=\mathbf{U}_{3} \sum \mathbf{U}_{3}^{*},
$$

where $\sum$ is a diagonal matrix consisting of the eigenvalues $\left(\lambda_{i}\right.$ $(i=1,2,3))$ of $\mathbf{T}_{3} ; \mathbf{U}_{3}^{*}$ is a complex conjugate transposition matrix; and $\mathbf{U}_{3}$ is an $\mathbf{U}$ matrix including the eigenvector of $\left[\mathbf{T}_{3}\right]$ [27].

Two important parameters (the scattering entropy and the antientropy) were defined by Cloude [28] in 1986 using the eigenvalues of the coherence matrix based on $\mathbf{T}_{3}$. The scattering entropy $(H)$ was obtained from the three eigenvalues as

$$
\begin{aligned}
& P_{i}=\frac{\lambda_{i}}{\sum_{j} \lambda_{j}}, \\
& H=\sum_{i=1}^{3}-P_{i} \log _{3}^{P_{i}} .
\end{aligned}
$$

From (4), the range of $H$ is $0-1$. The $H$ parameter can be regarded as the output of a stochastic calculation within a resolution unit, based on the backscattering mechanism. In other words, $H$ represents the probability of effective scattering. When $H$ approaches 0 , the main types of associated land surfaces are isotropically pure and mediate effective scattering. When $H$ is equal to 0 , there is one only nonzero eigenvalue in $\left[\mathbf{T}_{3}\right]$, with rank $1, \lambda_{2}=\lambda_{3}=0$. When $H$ approaches 1, random scattering is in play. When $H$ is equal to 1 , the three nonzero eigenvalues are equal [26]. When $H$ lies between 0 and 1 , the system changes from isotropic simple scattering to completely random scattering [28]. Therefore, $H$ also reflects coherent nondepolarization backscattering based on the object matrix. In fact, the $H$ values of most natural objects lie between 0 and 1 [26]. Empirically, when the $H$ value is $<0.3$, the scattering object is weakly depolarized, and the dominant scattering mechanism can be recovered based on the features of a specifically recognizable, equivalent point object. Here, the eigenvector corresponding to the maximum eigenvalue is selected and the others ignored. However, when $H$ is higher, the set of scattering objects is depolarized, and no single equivalent point object can be identified. Thus, the number of recognizable classes falls as $H$ increases [27].

The second important parameter is the antientropy $(A)$ value, defined as the value associated with normalization of the second and third object components [27]. A is given by

$$
A=\frac{P_{2}-P_{3}}{P_{2}+P_{3}}=\frac{\lambda_{2}-\lambda_{3}}{\lambda_{2}+\lambda_{3}} .
$$

$A$ also ranges from 0 to 1 and can be taken to complement $H$. A reflects the relative importance of the second and third eigenvalues and can also be viewed as a source of difference when $H$ is $>0.7$, as the second and third eigenvalues are greatly affected by noise when $H$ is less than this value [29]. Therefore, $A$ also represents the noise level. As $H$ increases, the types of ground objects recognized become fewer in number. Therefore, the use of $H$ alone will be inadequate. Then, $A$ can assist in recognition of the object type [28]. $A$ is particularly key in PolSAR applications. When $A$ reaches a maximum, $A$ can be used to distinguish scattering objects. Usually, when $A$ is higher, only the second scattering process is in play. When $A$ is lower, both scattering processes are equally strong. Therefore, a combination of $H$ and $A$ parameters greatly aids in solving polarization scattering problems because the eigenvalues of the $\mathbf{U}$ matrix are invariant. Using the $A$ parameter, the object scattering information imparted by the three eigenvalues is evident on polarization spectra created using different combinations of $H$ and $A$ [26]. The four such spectra are given by

$$
S \Rightarrow\left\{\begin{array}{l}
(1-H) *(1-A) \\
H *(1-A) \\
A *(1-H) \\
H * A .
\end{array}\right.
$$

These spectra, termed polarization characteristic decomposition spectra [26], contain all "random" information about the scattering object. The satellite polarization electromagnetic power can be divided into four parts by reference to the spectra, and each is a component of the scattering mechanism.

The scattering angle is the third relevant parameter; this represents the type of scattering mechanism in play. The angle is defined as

$$
\alpha=p_{1} * \alpha_{1}+p_{2} * \alpha_{2}+p_{3} * \alpha_{3} .
$$

When $\alpha$ approaches $\pi / 4$, this represents the volume scattering component; when $\alpha$ is $\pi / 2$, this represents the dihedral scattering component; and when $\alpha$ is 0 , this represents the odd scattering component $[29,30]$.

3.2. The Freeman-Durden Method. The three-component Freeman-Durden polarization decomposition method was developed in 1998 [31, 32]. The method differs from the H/A/Alpha method. The surface, dihedral, and volume scattering components are obtained directly from a scattering coherent matrix [33]. The surface scattering component contains only ground soil information (thus, no vegetation information) [34]. Therefore, the scattering information from vegetation and soil is completely separated. In other words, the method eliminates the vegetation effects on the SMC. The coherent matrices differ for different surface scattering models [34]. Here, we used the Bragg and $\mathrm{X}$-Bragg models. The Bragg model can be viewed as a special case of the X-Bragg model. The X-Bragg model is an improved Bragg model, resolving the problems of decomposition and nonzero cross-polarization [35]. When $\delta=0$, the Bragg model is equivalent to the X-Bragg model [33]. The coherent matrices are as follows: 


$$
\begin{aligned}
& {\left[\mathbf{T}_{\mathrm{Bragg}}\right]=f_{\mathrm{S}}\left[\begin{array}{ccc}
1 & \beta^{*} & 0 \\
\beta & |\beta|^{2} & 0 \\
0 & 0 & 0
\end{array}\right]+f_{\mathrm{D}}\left[\begin{array}{ccc}
|\alpha|^{2} & \alpha & 0 \\
\alpha^{*} & 1 & 0 \\
0 & 0 & 0
\end{array}\right]+\frac{f_{\mathrm{V}}}{4}\left[\begin{array}{lll}
2 & 0 & 0 \\
0 & 1 & 0 \\
0 & 0 & 1
\end{array}\right]} \\
& {\left[\mathbf{T}_{\mathrm{X}-\mathrm{Bragg}}\right]=f_{\mathrm{S}}\left[\begin{array}{ccc}
1 & \beta^{*} \sin c(2 \delta) & 0 \\
\beta \sin c(2 \delta) & \frac{1}{2}\left|\beta^{2}\right|(1+\sin c(4 \delta)) & 0 \\
0 & 0 & \frac{1}{2}\left|\beta^{2}\right|(1-\sin c(4 \delta))
\end{array}\right]+f_{\mathrm{D}}\left[\begin{array}{ccc}
\left|\alpha^{2}\right| & \alpha & 0 \\
\alpha^{*} & 1 & 0 \\
0 & 0 & 0
\end{array}\right]+f_{\mathrm{V}}\left[\begin{array}{ccc}
2 & 0 & 0 \\
0 & 1 & 0 \\
0 & 0 & 1
\end{array}\right]}
\end{aligned}
$$

where $\left[\mathbf{T}_{\mathrm{Bragg}}\right]$ and $\left[\mathbf{T}_{\mathrm{X}-\mathrm{Bragg}}\right]$ are the Bragg and X-Bragg coherent matrices; $f_{\mathrm{S}}, f_{\mathrm{D}}$, and $f_{\mathrm{V}}$ are the scattering amplitudes of the odd, dihedral, and volume components, respectively; $\beta$ and $\alpha$ are the odd and dihedral scattering parameters; and $\delta$ is the extent of depolarization [36].

\section{Results and Discussion}

4.1. Backscattering of Typical Objects. The backscattering characteristics of different objects differ; this is of fundamental importance in terms of image classification using SAR data. It is important to analyze the backscattering characteristics of different objects when simulating backscattering coefficients.

We chose four typical objects and analyzed the spatiotemporal changes in backscattering coefficients; the objects were bare soil fields, maize fields, orchards, and cotton fields. Figure 1 shows the 27 random sample points (10 in maize fields, 9 in orchards, and 8 in cotton fields) and 22 points along tree-lined roads. GF-1 data were used as the auxiliary data to distinguish land cover types. We evaluated changes in backscattering coefficients of Radarsat-2 data for different objects, such as maize field, bare field, and orchard (Figures 3-6, 20140627: the sowing period of maize, 20140721: the jointing period of maize, 20140814: the heading period of maize, and 20140907: the flowering period of maize).

Figure 3 shows that the C-band backscattering coefficients of maize changed over time. The $\mathrm{HH}$ coefficient fell as maize grew, peaking at about $-5 \mathrm{~dB}$ at the jointing stage on July 21, 2014. During flowering, the rate of change was reduced and then ceased. Thus, the C-band coefficient was greatly affected by maize growth. After the heading stage, it is almost impossible to obtain soil surface backscattering coefficients using C-band Radarsat- 2 data. Thus, it is essential to reduce the effects of vegetation after heading. The $\mathrm{HH}$ and $\mathrm{VV}$ coefficients ranged from -4 to $-16 \mathrm{~dB}$, and those of $\mathrm{HV}$ and $\mathrm{VH}$ from -10 to $35 \mathrm{~dB}$. The $\mathrm{HH}$ or $\mathrm{VV}$ changes (about $16 \mathrm{~dB}$ ) were less than those of HV or VH (about $20 \mathrm{~dB}$ ). Therefore, when we compared the changes in the four polarization backscattering coefficients, those of $\mathrm{HV}$ and $\mathrm{VH}$ were more obvious and coherent than those of $\mathrm{HH}$ and $\mathrm{VV}$ at all sampling times. Changes in $\mathrm{HH}$ or VV backscattering were reflected by a simple cubic regression equation with a correlation coefficient of approximately unity ( $\mathrm{HV}$ in Figure 3). This equation showed the relationship between the backscattering coefficient of SAR data and maize growth periods. This regression equation indicated how the backscattering coefficients of the C-band Radarsat-2 sensors changed, approximately simulating backscattering at various maize growth stages. With the growth of maize, the $\mathrm{HV}$ backscattering coefficient of SAR data is descending in Figure 3. This afforded the theoretical support required for image classification using the $H / A$ /Alpha polarization decomposition method of C-band Radarsat- 2 data.

Figure 4 shows that there is no change rule of the C-band $\mathrm{HH}$ and VV backscattering coefficients of orchards. As maize grew, $\mathrm{HV}$ and $\mathrm{VH}$ backscattering decreased. In particular, HV backscattering ranged from about -25 to $-5 \mathrm{~dB}$, a range of $20 \mathrm{~dB}$. Figure 5 shows that the cotton backscattering changes were similar to those of maize. Therefore, maize and cotton cannot be distinguished using only backscattering coefficients; furthermore, those of both crops changed with growth. Thus, maize and cotton fields can be divided into only bare soil, full-cover vegetation field (vegetation field), and mixed fields. However, Figure 6 shows that the backscattering coefficients of tree-lined roads obviously changed with tree growth. On June 27, 2014, the $\mathrm{HH}$ and VV backscattering coefficients ranged from 0 to $-20 \mathrm{~dB}$. Thereafter, the $\mathrm{HH}$ or $\mathrm{VV}$ ranged from -4 to $-12 \mathrm{~dB}$; the maximal variation fell from 20 to $8 \mathrm{~dB}$, indicating that roadside trees did not change much from July to September. The changes in $\mathrm{HV}$ or VH backscattering coefficients were similar to those of $\mathrm{HH}$ or VV.

The backscattering characteristics of typical objects over time and space are shown in Figures 3-6. The backscattering characteristics of maize and cotton clearly changed as the crops grew, and this change could be expressed by regression equations. Maize and cotton cannot be distinguished by backscattering coefficients alone, but we show below that maize and cotton fields can be distinguished in the threefield state (bare soil, full-cover (maize and cotton), and mixed). The advantage of this approach is that the vegetation type is unimportant, allowing retrieval of SMC data via polarization decomposition of C-band Radarsat-2 data. 


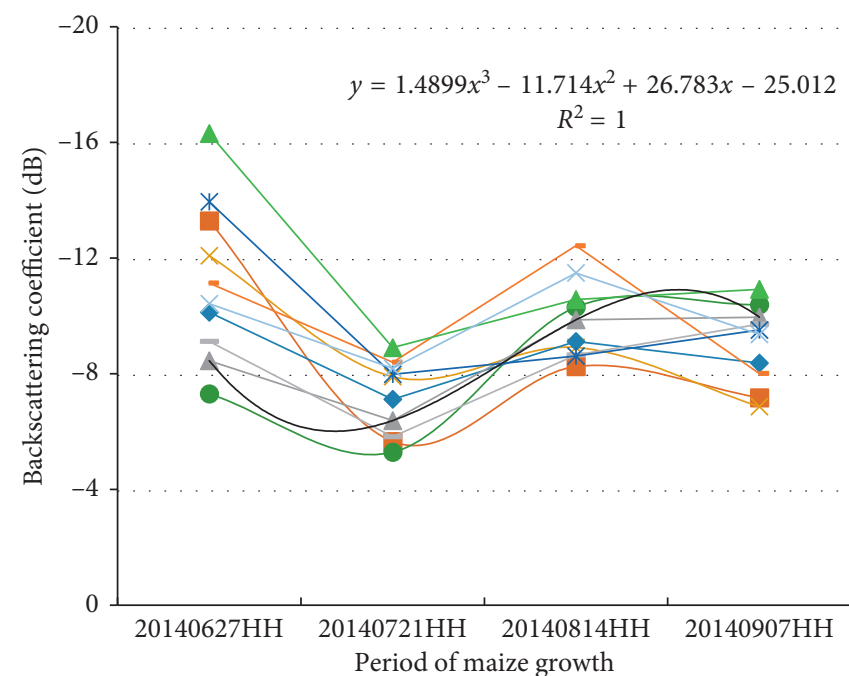

(a)

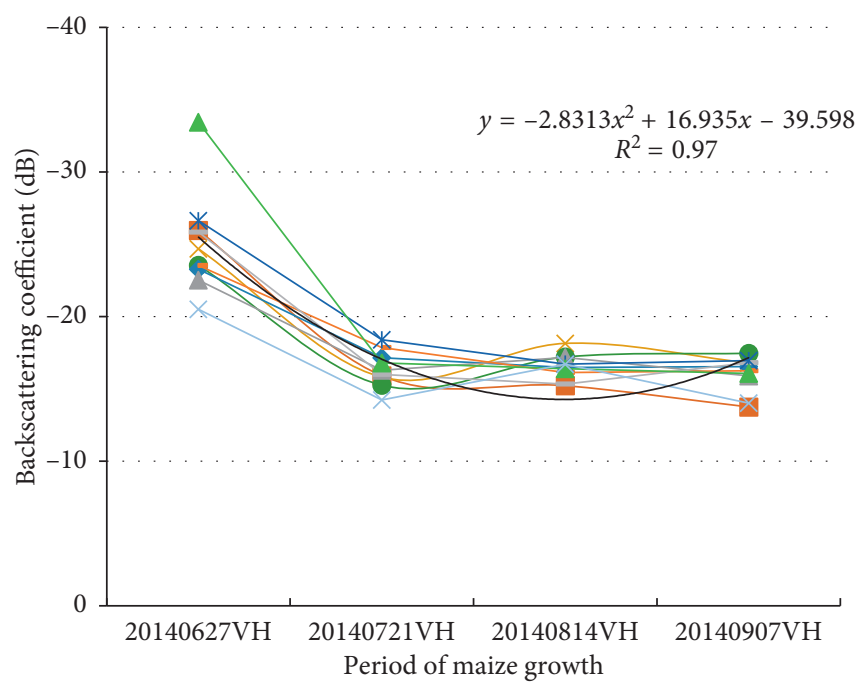

(c)

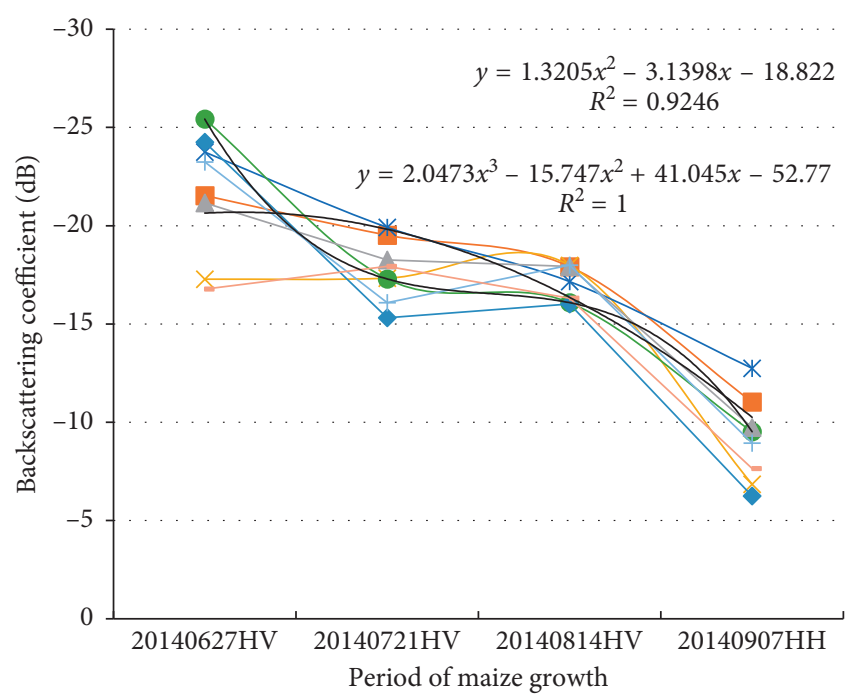

(b)

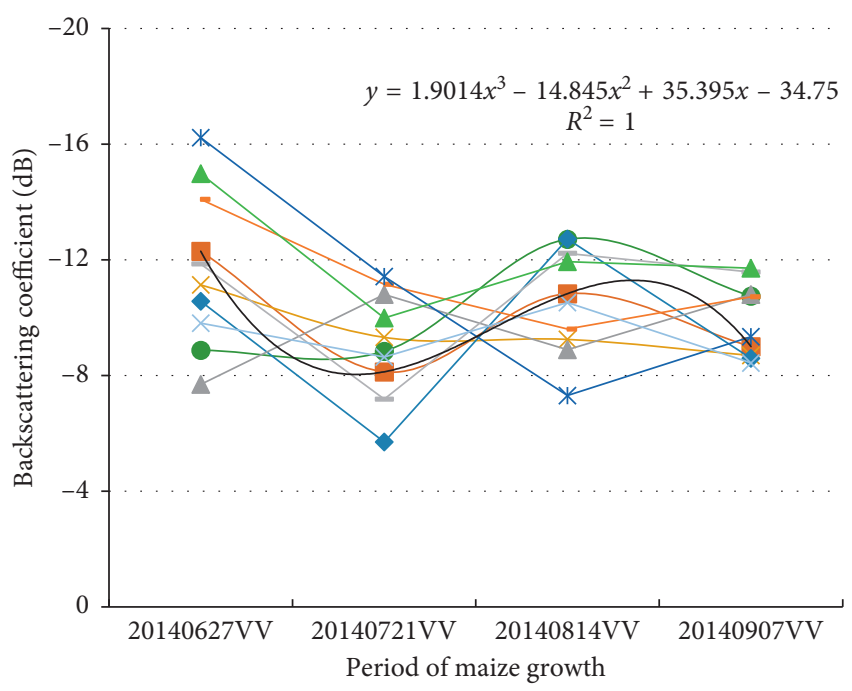

(d)

Figure 3: Backscattering coefficients used to evaluate maize fields.

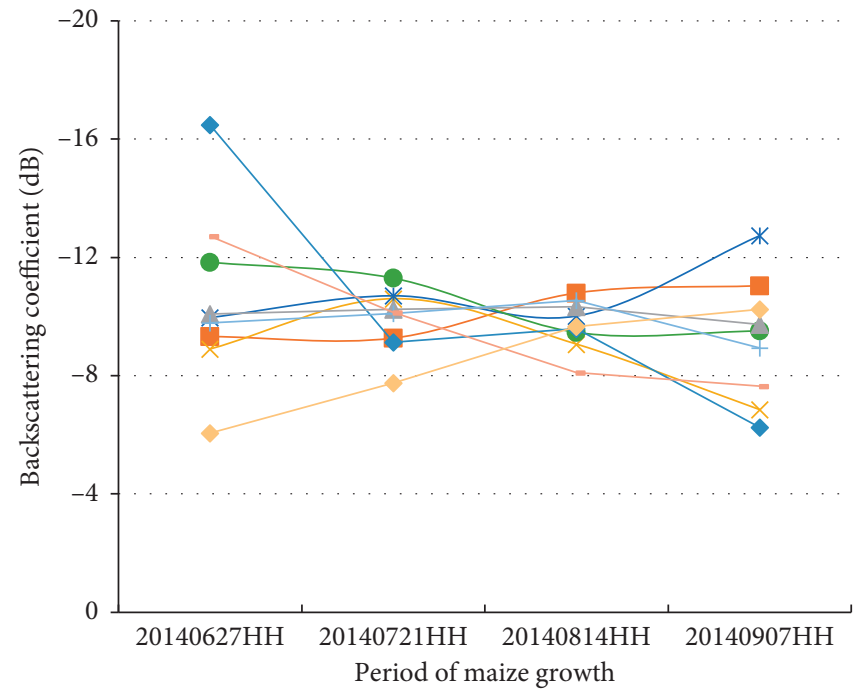

(a)

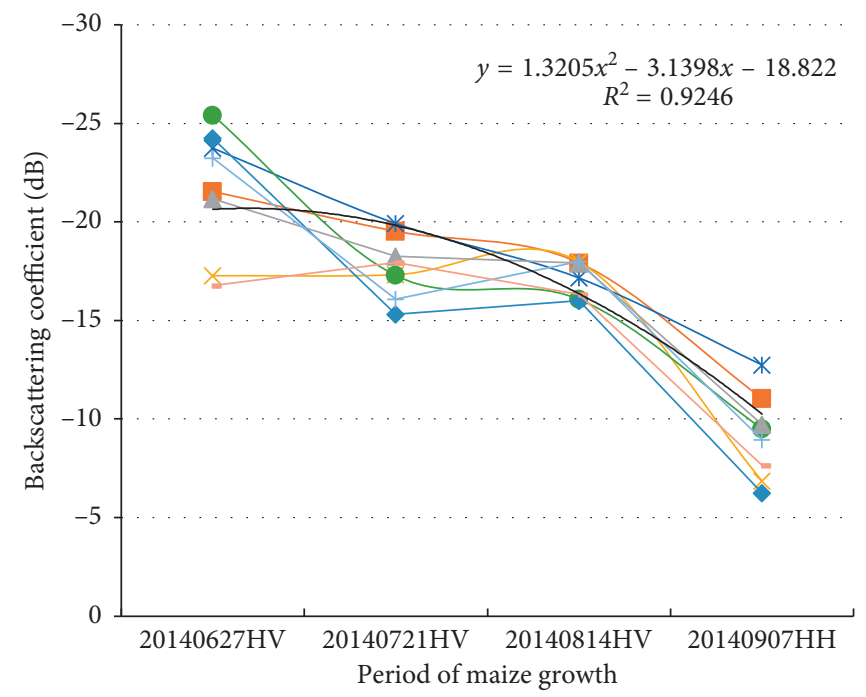

(b)

Figure 4: Continued. 


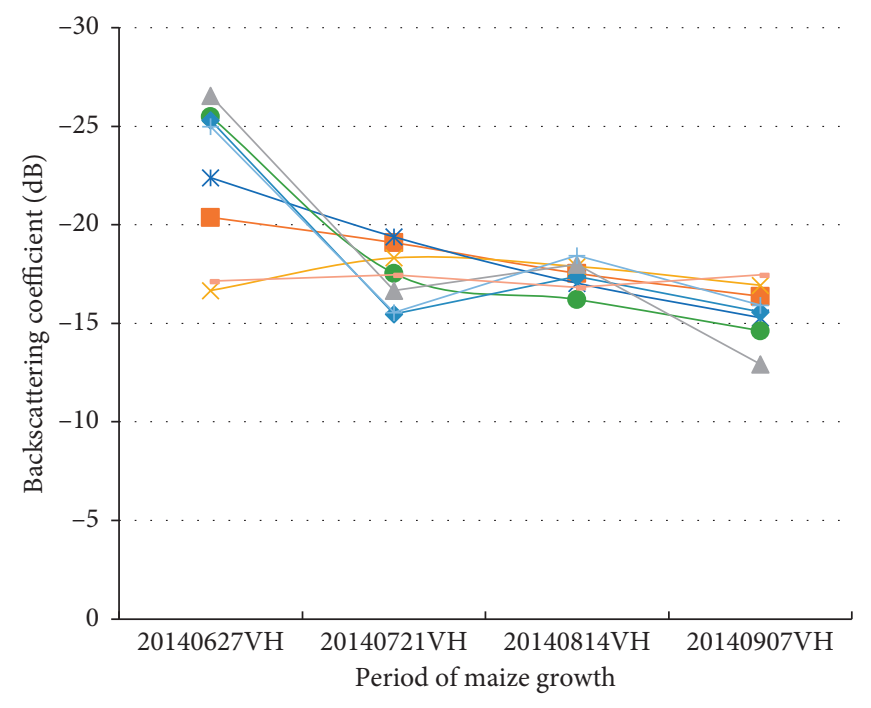

(c)

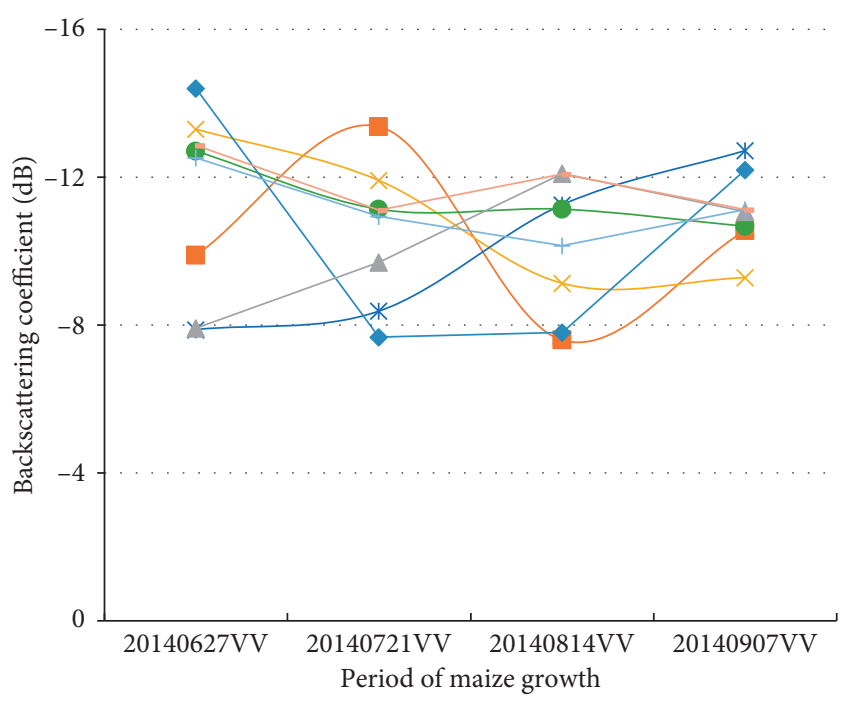

(d)

FiguRE 4: Backscattering coefficients used to evaluate orchards.

4.2. Thresholds of Typical Objects. To optimize SMC estimates, the retrieval methods differ by surface types. By reference to the backscattering characteristics of different objects, namely, bare soil fields, vegetation, mixed fields, and urban/city areas, we used color composites of the June 2014 Radarsat-2 data to this end (Figure 7). We derived the twodimensional feature spaces of $H$ and the Alpha decompositional components of these four surface cover types (Figure 8).

Figure 8 shows that the feature space is occupied by the $H$ and Alpha decompositional components of the various surface cover types. Differences in surface cover can generally be distinguished by their ranges of $H$ and Alpha values. The bare soil sample points (red) cluster at the lower left of the feature space. Vegetation sample points (gray) cluster at the upper right. Urban/city sample points (yellow) cluster at the upper left. The bare soil and vegetation sample points are closer to each other than the urban/city points. In particular, a distributional rule controlling sample point mixing (orange points) is evident. As vegetation increases, the distribution also increases in a manner that can be expressed by a simple regression equation with a correlation coefficient of about 0.9. This equation showed the relationship between $H$ and alpha parameters. By this equation, there is a change in the characteristic that $H$ and alpha are increasing from the bare soil to mixing to vegetation. The distributional differences among surface cover types support the image classification afforded by the $H / A$ /Alpha polarization decomposition method and lay the foundation for the improved SMC retrieval method that follows.

We used different thresholds to distinguish different surface covers. In order to obtain the thresholds of different land cover types, the histograms of different surface covers were used to estimate thresholds. Histograms showing the features of different surface covers are shown in Figure 9. As not all pixels of the sample area are pure, we also show the normal distributions of the $H$ and Alpha decomposition components. The histogram distributions are closer (more concentrated) in the sampled areas, and the numbers of pure pixels are higher. Here, threshold values were determined by reference to $H$ or Alpha decomposition component frequencies $>100$, except in urban/city areas. Therefore, the $H$ and alpha thresholds of different surface covers can be obtained.

Figure 9 shows that the $H$ and Alpha threshold values of bare soil fields were $0.18-0.42$ and $7-24^{\circ} \mathrm{C}$, respectively. The peak $H$ and Alpha values were 0.3 and $15^{\circ} \mathrm{C}$. The $H$ and Alpha values of vegetated fields were $0.61-88$ and $22-50^{\circ} \mathrm{C}$, respectively. The peak $H$ and Alpha values were 0.65 and $40^{\circ} \mathrm{C}$. Bare and vegetated fields could be distinguished by their $H$ and Alpha threshold values. However, the $H$ and Alpha threshold values of urban/city areas were $0-1$ and $18-90^{\circ} \mathrm{C}$, respectively. The $H$ and Alpha threshold values of mixed fields were $0.2-0.9$ and $9-25^{\circ} \mathrm{C}$, respectively. The peak $H$ and Alpha values were 0.55 and $22^{\circ} \mathrm{C}$. These ranges were rather wide. The thresholds of mixed fields and urban/city areas overlapped with those of bare soil and vegetation fields (Table 2).

The main scattering mechanism in play in bare soil areas was surface scattering. In contrast, the principal scattering mechanism in vegetated areas was volume scattering. The $H$ and Alpha threshold values of bare soils and vegetated fields allowed the surface and volume mechanisms to be distinguished, affording a useful foundation for SMC retrieval based on the Freeman-Durden polarization decomposition method.

4.3. Image Classification. To retrieve SMC, we used a polarization decomposition technique to decompose the backscattering coefficients of Radarsat- 2 into three components: surface, volume, and dihedral scattering. In this study, the two classification methods (maximum-likelihood 


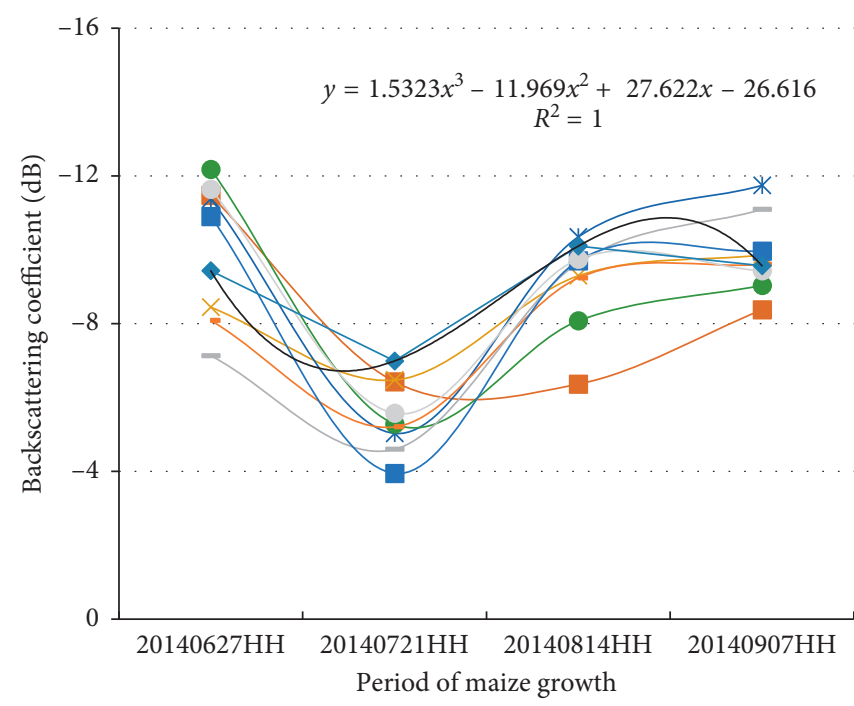

(a)

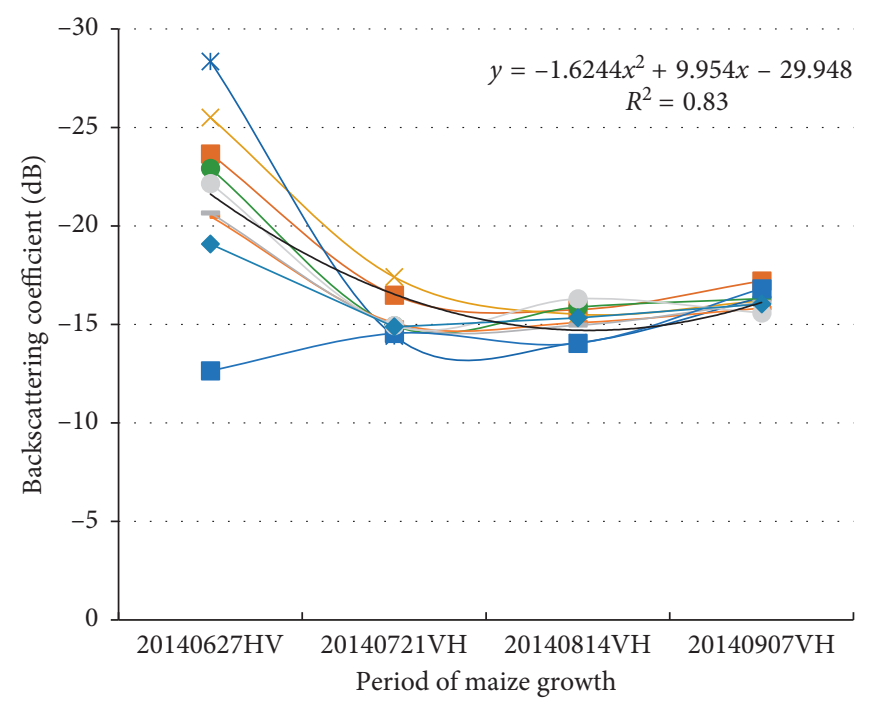

(c)

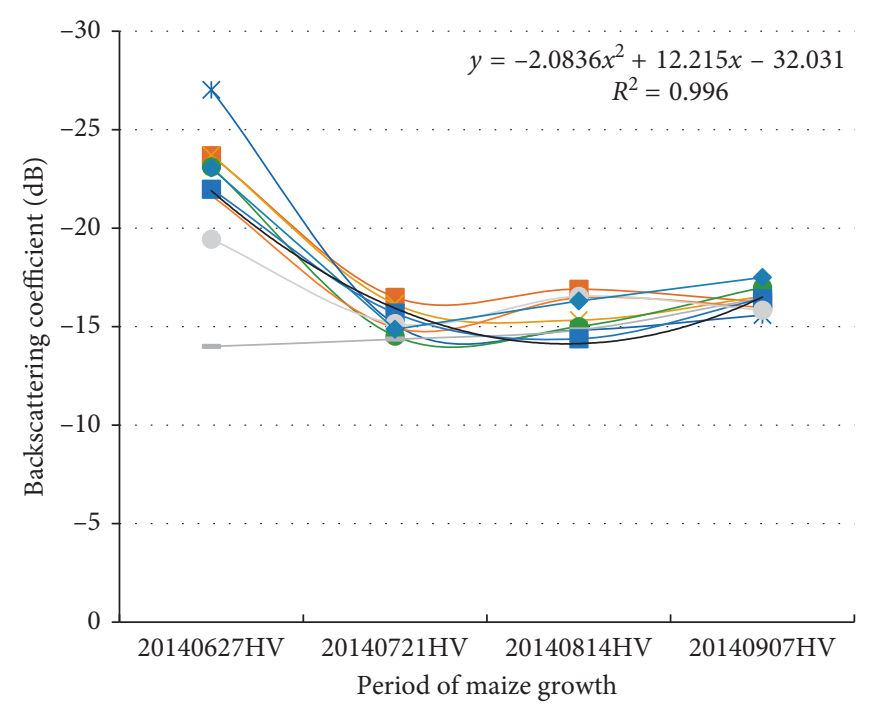

(b)

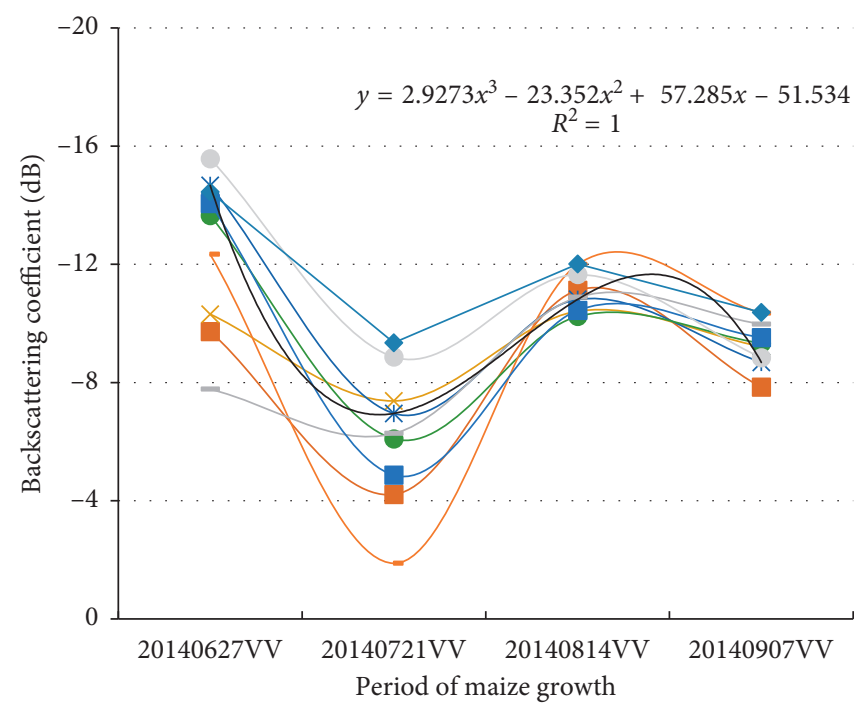

(d)

Figure 5: Backscattering coefficients used to evaluate cotton fields.

and threshold classification) were used to acquire the three components (Figure 10). The maximum-likelihood classification is a statistical method employed to calculate correlation probability density functions. This method is commonly used in remote sensing. However, the regions of interest are manually selected. The threshold classification method is more objective; thresholds are determined by the feature spaces of $H$ and Alpha, thus reflecting information characteristics of different objects (Figure 8).

Figure 10 shows that the three components differed as maize grew. The classifications differed in terms of their spatial components. Generally, the proportion of the surface scattering component in the maximum-likelihood method was higher than that of the threshold classification. As maize grew, the surface scattering proportion decreased gradually. On August 14 and September 7, the surface scattering proportions were close to 0. Apart from the dihedral scattering of urban/city areas, volume scattering was almost exclusively in play. On July 21 , the dihedral scattering proportion of the maximum-likelihood classification was lower than that of the threshold classification. In reality, some mixed areas were evident between low-growing maize and bare soil surfaces. In such areas, maize stalks and bare soil readily formed dihedral corner reflectors. The threshold classification is then more appropriate.

4.4. Soil Moisture Retrieval. To retrieve SMCs as maize grew, we used the Freeman-Durden method to obtain three backscattering components: surface, dihedral, and volume. These components were then refined using the data of Figure 10. Finally, we used the Bragg and X-Bragg models to calculate soil dielectric constants and the Dobson model to 


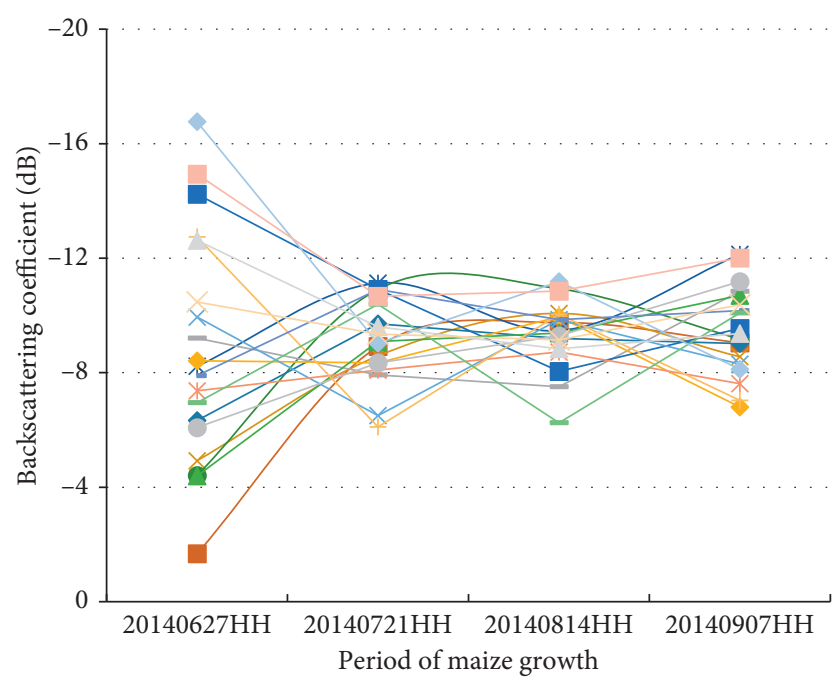

(a)

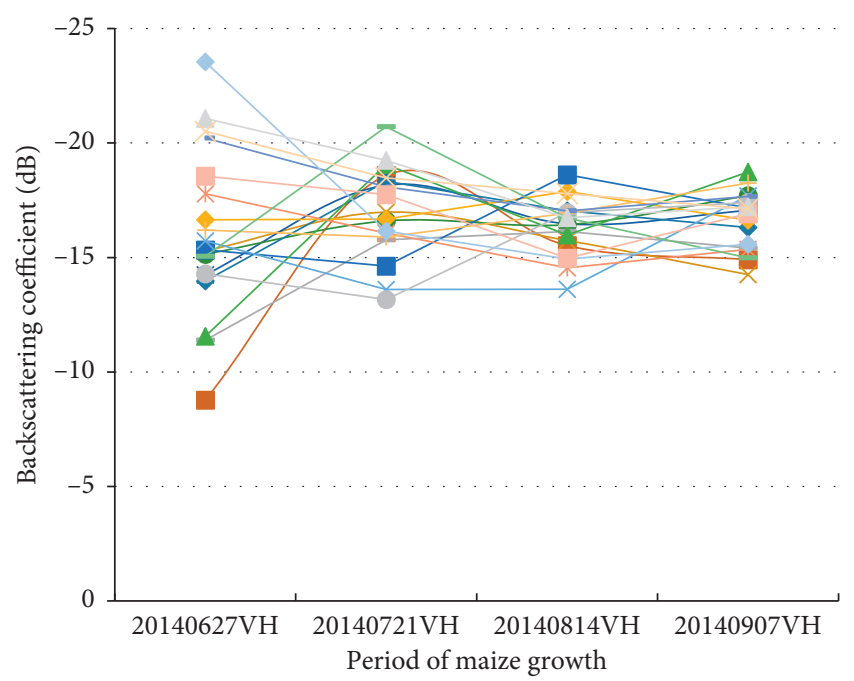

(c)

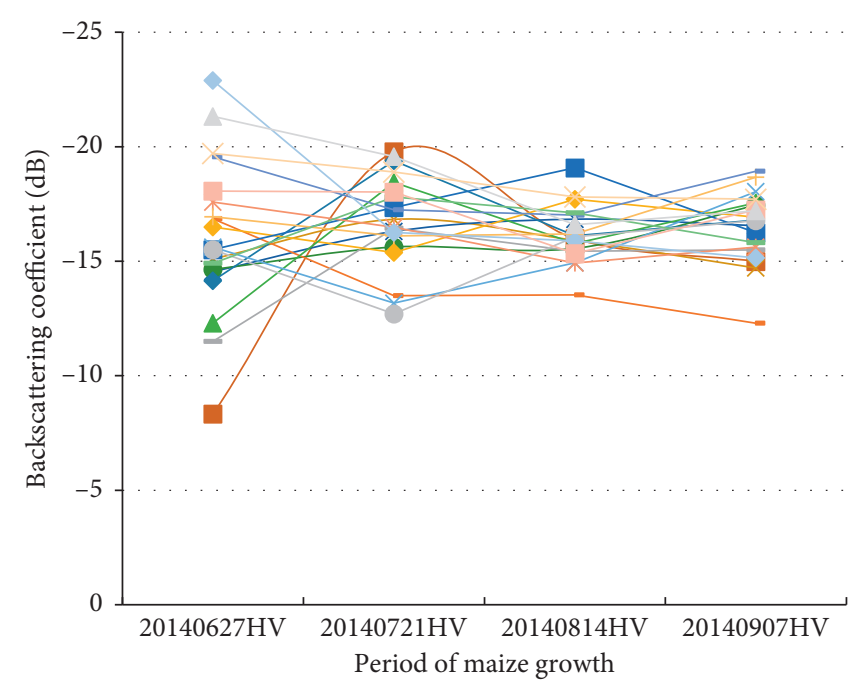

(b)

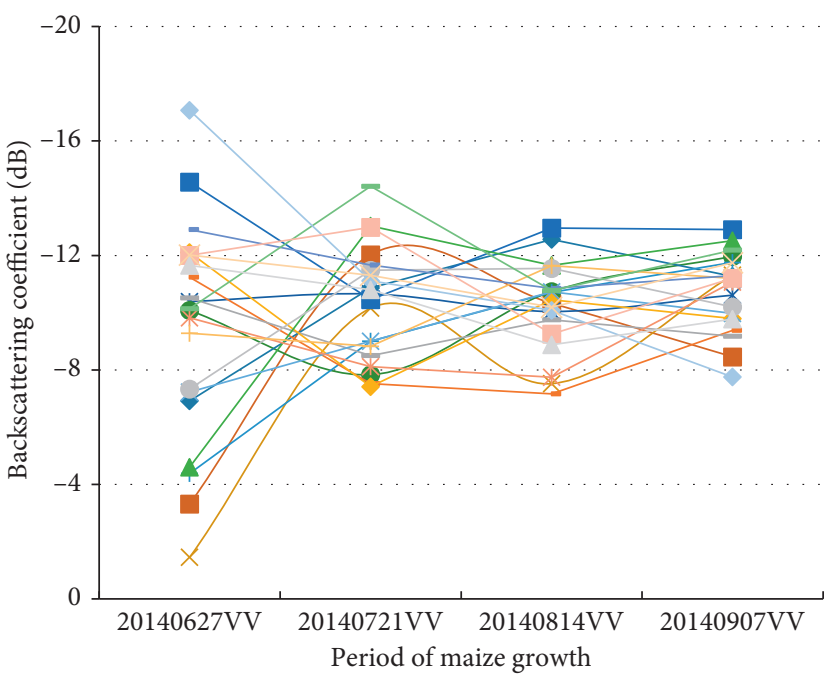

(d)

Figure 6: Backscattering coefficients used to evaluate roads bordered by trees.

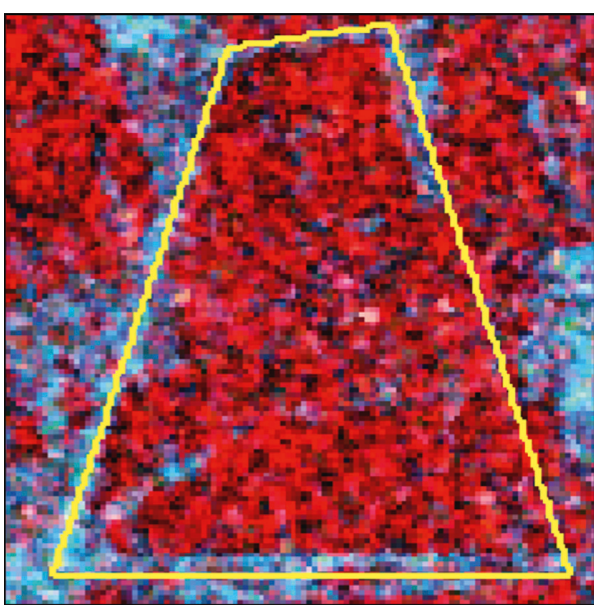

(a)

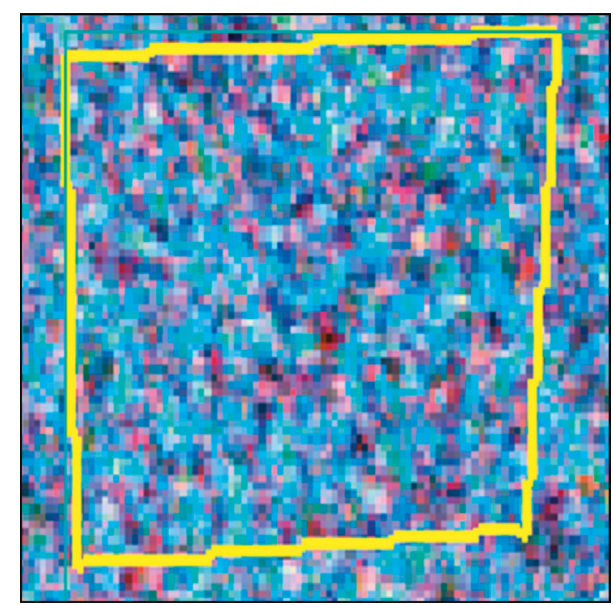

(b)

Figure 7: Continued. 


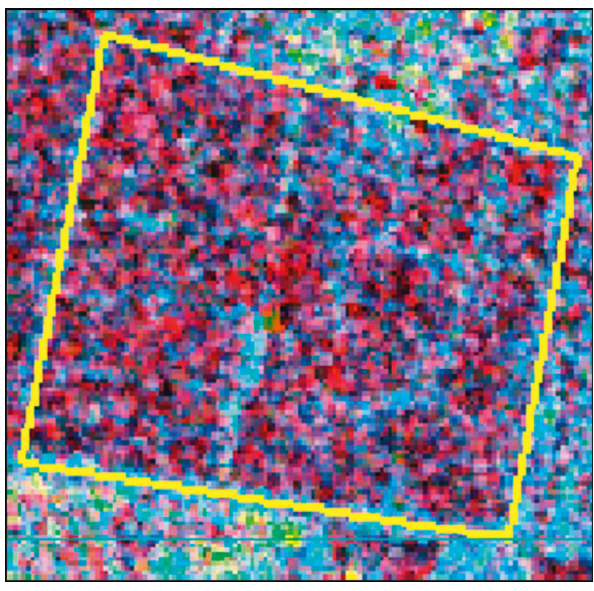

(c)

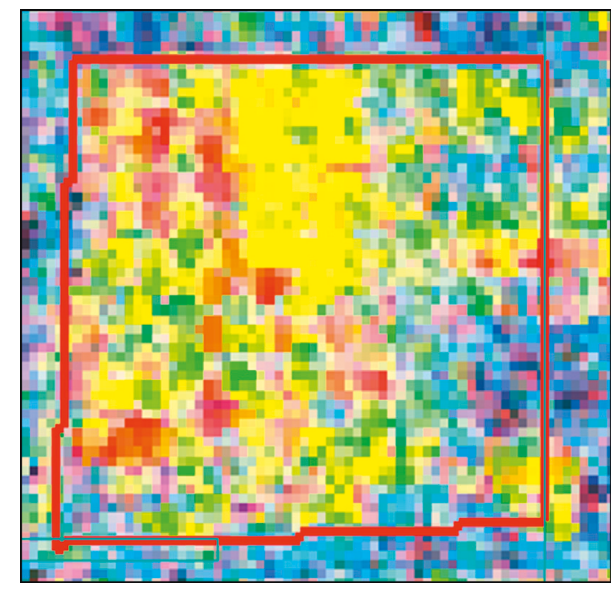

(d)

Figure 7: Typical examples of the four types of area examined using H/A/Alpha composite color images based on June 2014 Radarsat-2 data (H: red layer; A: green layer; Alpha: blue layer). (a) Bare soil, (b) vegetation, (c) mixing, and (d) urban/city.

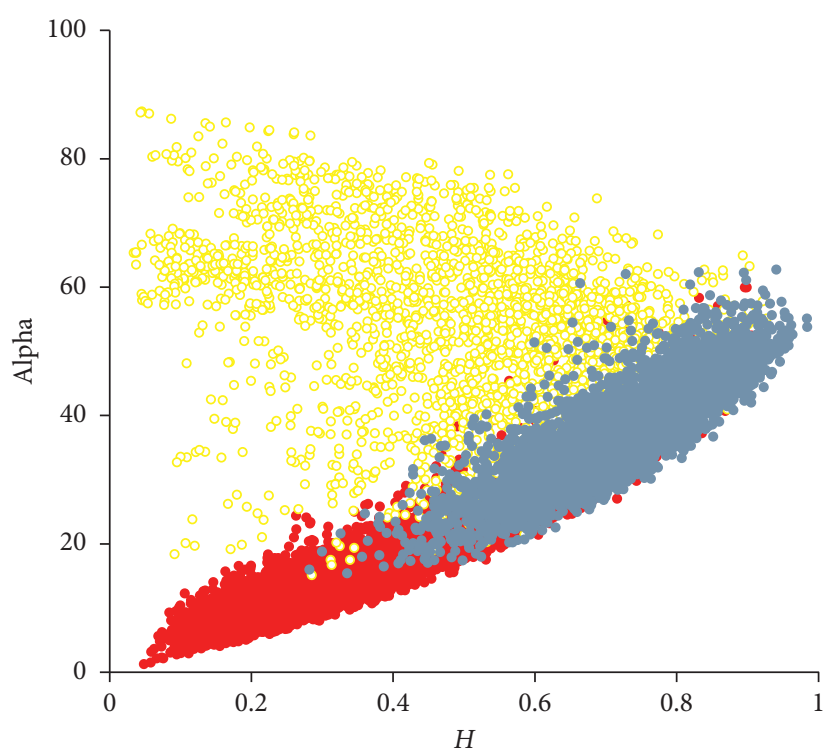

- Bare soil

Urban and city

- Vegetation

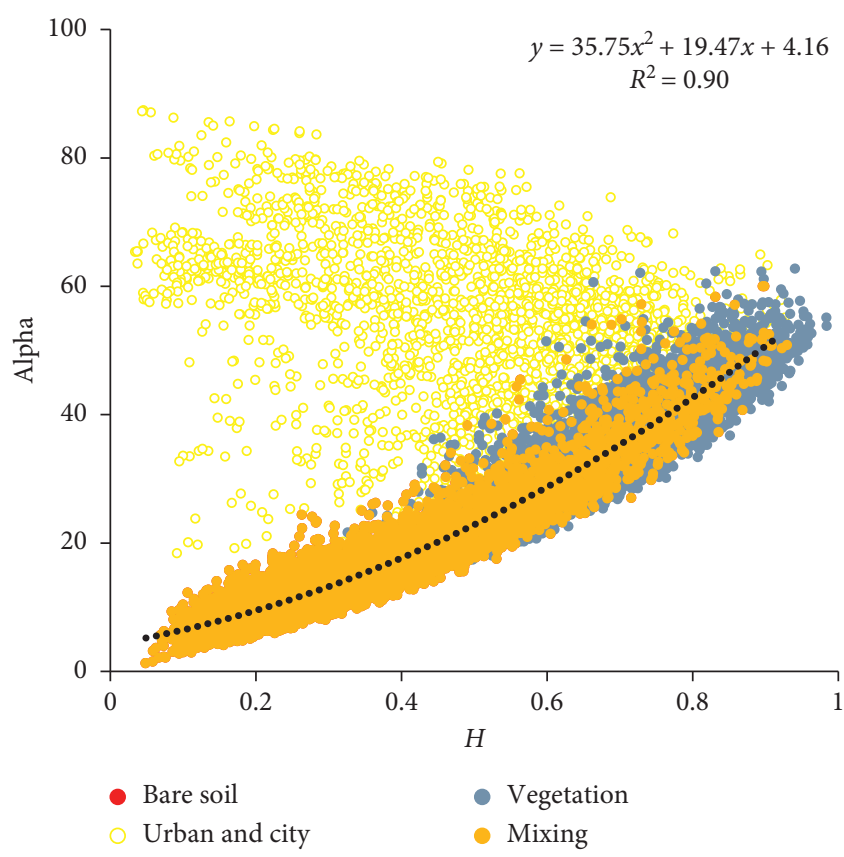

(b)

Figure 8: Two-dimensional feature space plots of $H$ and Alpha (red points: bare soil field; yellow points: vegetated field; orange points: a field with mixed bare soil and vegetation).

estimate soil moisture. We then calculated RMSEs to evaluate data accuracy (Figure 11).

Figure 11 shows that the X-Bragg soil moisture data retrieved on June 27, 2014, were the most accurate of all data obtained during the maize growth period (thus associated with the lowest RMSE value, 0.028). Overall, the X-Bragg model was better than the Bragg model at all maize growth stages. From July to September 2014, the data were less reliable because, as maize grew, moisture signals from the surface soil become increasingly difficult to obtain. Also, the C-band penetration of Radarsat-2 is limited; soil moisture data cannot be reliably retrieved using C-band microwave data, especially in the late stages of maize growth. Although the results are thus variable, our concept is valuable. The L-band penetrated the soil better than did the C-band. Our method can thus be used to retrieve soil moisture data delivered by the L-band.

\section{Conclusions}

When retrieving soil moisture data employing decomposition technology, it is essential that the three 

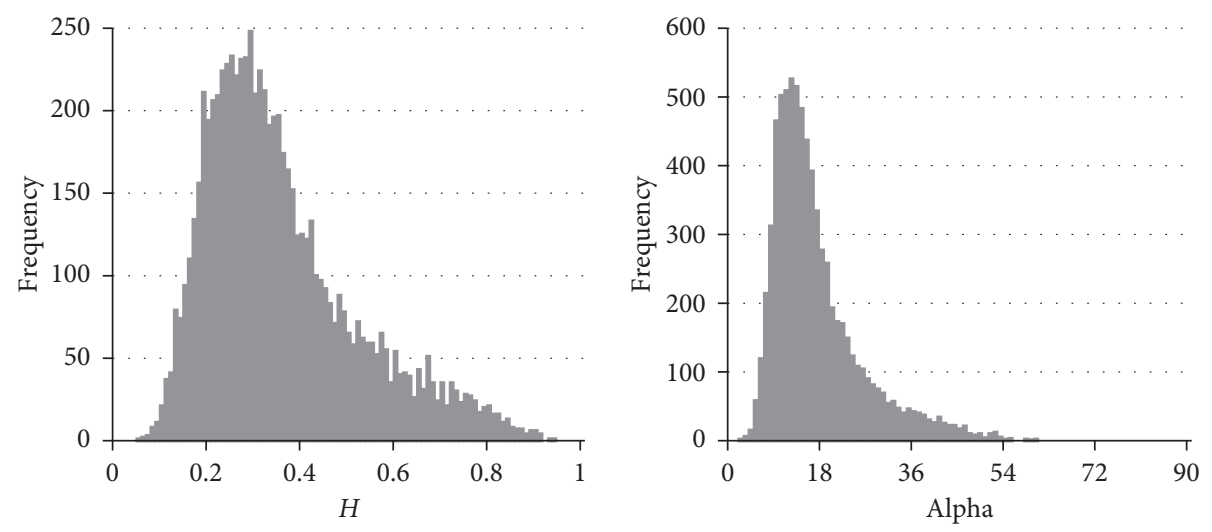

(a)
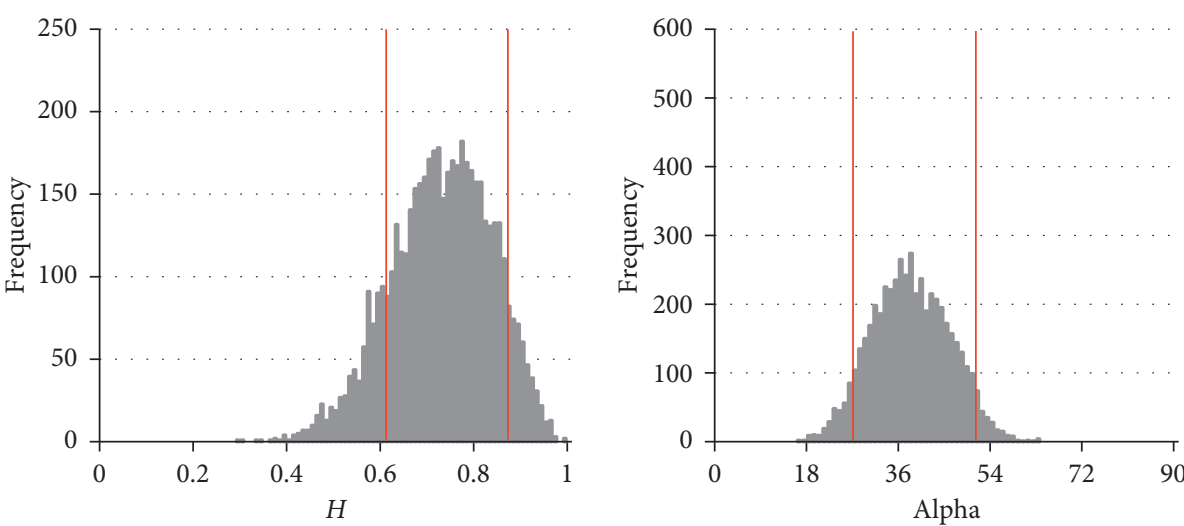

(b)
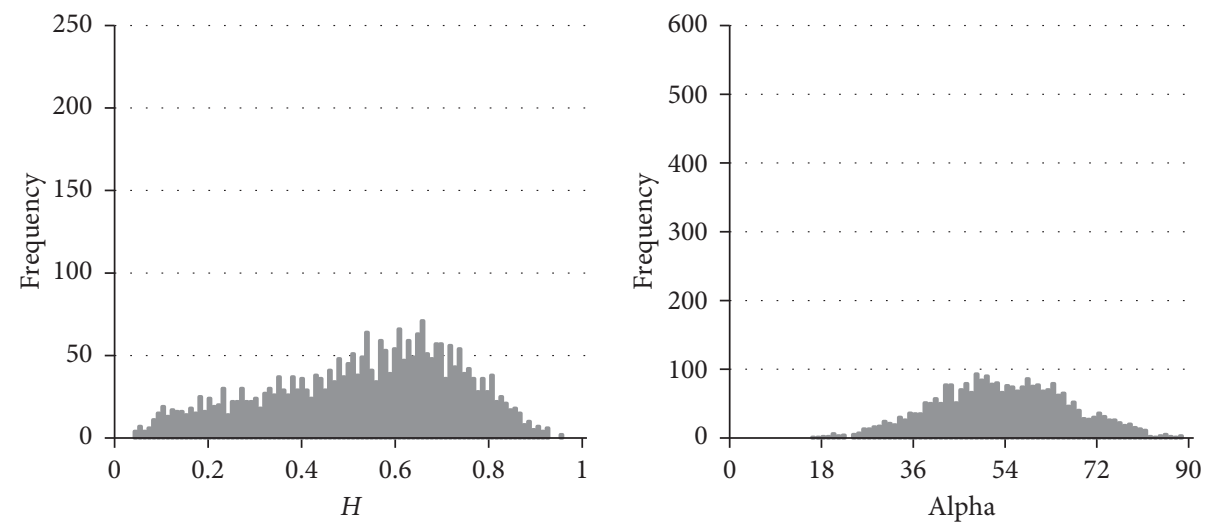

(c)
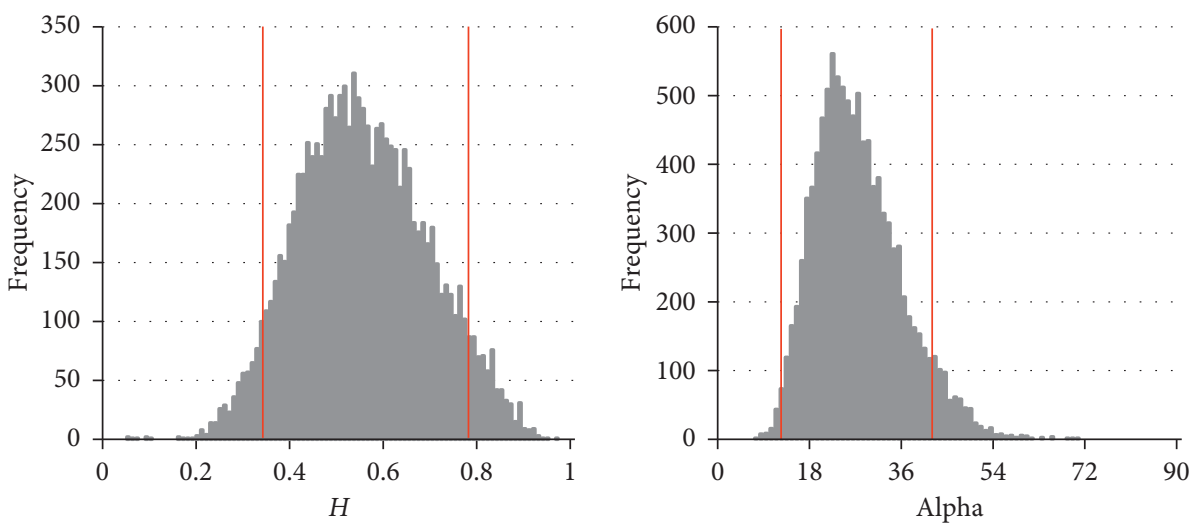

(d)

FiguRE 9: Histograms of $H$ and Alpha for four typical types of surface cover. (a) A bare soil field; (b) a vegetated field; (c) an urban/city area; (d) a field with mixed bare soil and vegetation. 
TABLE 2: Values obtained when evaluating surfaces differing in terms of cover.

\begin{tabular}{|c|c|c|c|c|c|c|c|c|c|c|}
\hline Types & H_min & H_max & H_mean & al_min $\left({ }^{\circ} \mathrm{C}\right)$ & al_max $\left({ }^{\circ} \mathrm{C}\right)$ & al_mean $\left({ }^{\circ} \mathrm{C}\right)$ & H_th & al_th $\left({ }^{\circ} \mathrm{C}\right)$ & H_p & al_p $\left({ }^{\circ} \mathrm{C}\right)$ \\
\hline The bare soil field & 0.049 & 0.930 & 0.350 & 1.27 & 59.96 & 16.32 & $0.2-0.4$ & $7-24$ & 0.3 & 15 \\
\hline The vegetation field & 0.282 & 0.984 & 0.720 & 15.40 & 62.70 & 37.45 & $0.6-0.9$ & $22-50$ & 0.7 & 40 \\
\hline The urban/city field & 0.034 & 0.957 & 0.526 & 15.10 & 97.34 & 52.47 & $0-1$ & $18-90$ & 0.65 & 50 \\
\hline The mixing field & 0.041 & 0.973 & 0.544 & 6.65 & 69.09 & 26.48 & $0.2-1$ & $9-50$ & 0.5 & 25 \\
\hline
\end{tabular}

$H \_$min: $H$ minima of sample areas; $H \_$max: $H$ maxima; $H \_$mean: $H$ mean values; al_min: Alpha minima; al_max: Alpha maxima; al_mean: Alpha mean values; $H \_$th: $H$ threshold ranges; al_th: Alpha threshold ranges; $H \_p: H$ peak values; al_p: Alpha peak values.

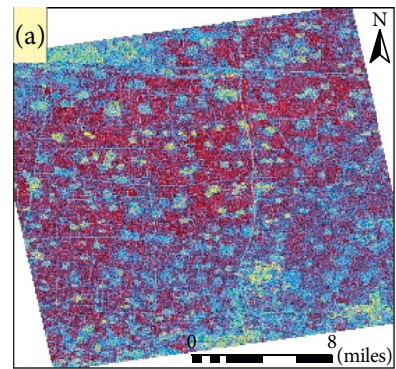

$2014 / 06 / 27$

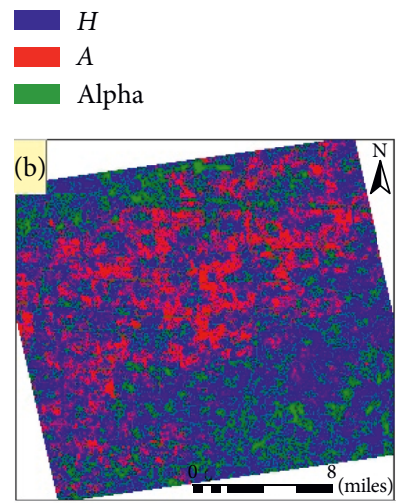

$2014 / 06 / 27$

Dihedral scattering

Old scattering

Volume scattering

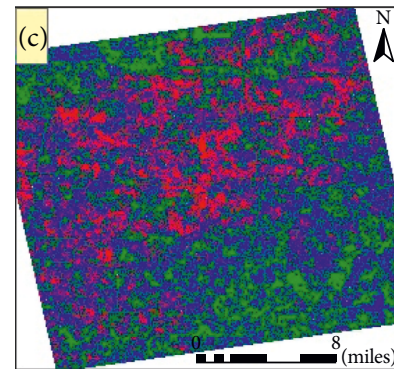

2014/06/27

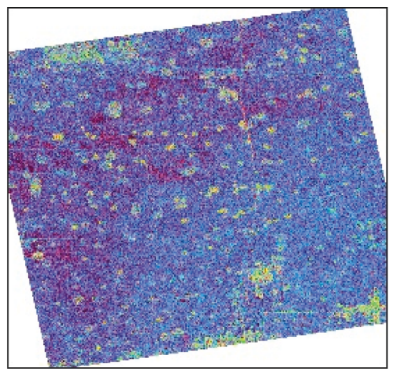

$2014 / 07 / 21$

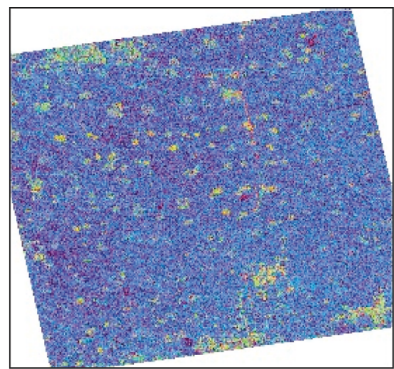

2014/08/14

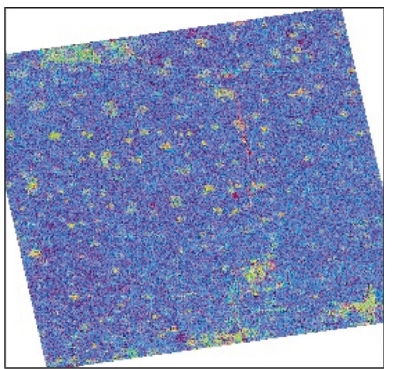

2014/09/07

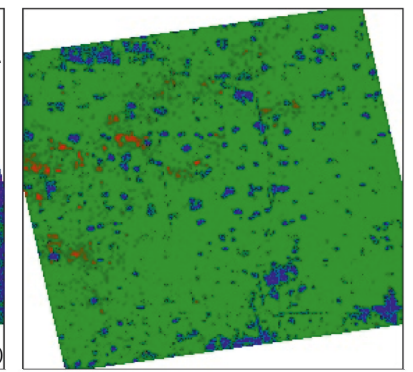

$2014 / 07 / 21$

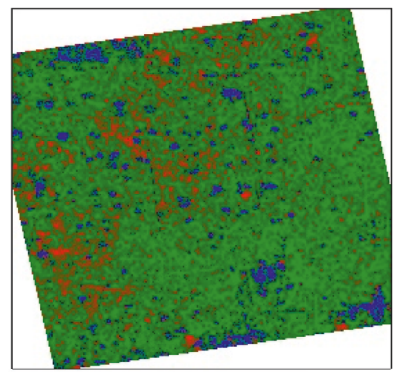

2014/08/14

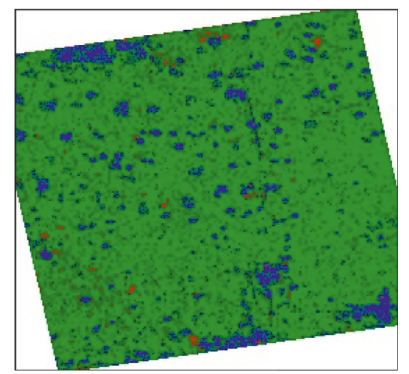

2014/09/07

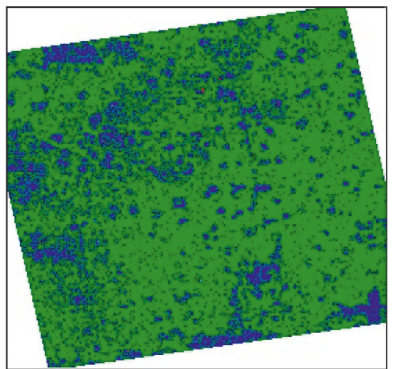

2014/07/21

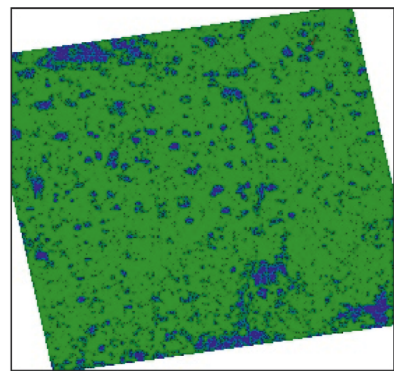

2014/08/14

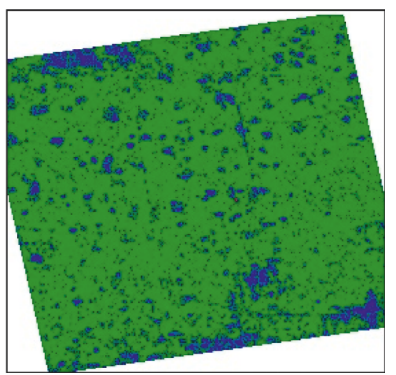

2014/09/07

Dihedral scattering

Old scattering

Volume scattering

FIgURE 10: Classification of images based on the H/A/Alpha decomposition method. (a) Synthetic color images of $H$, $A$, and Alpha. (b) Images obtained using the maximum-likelihood method. (c) Images obtained using the threshold method. 


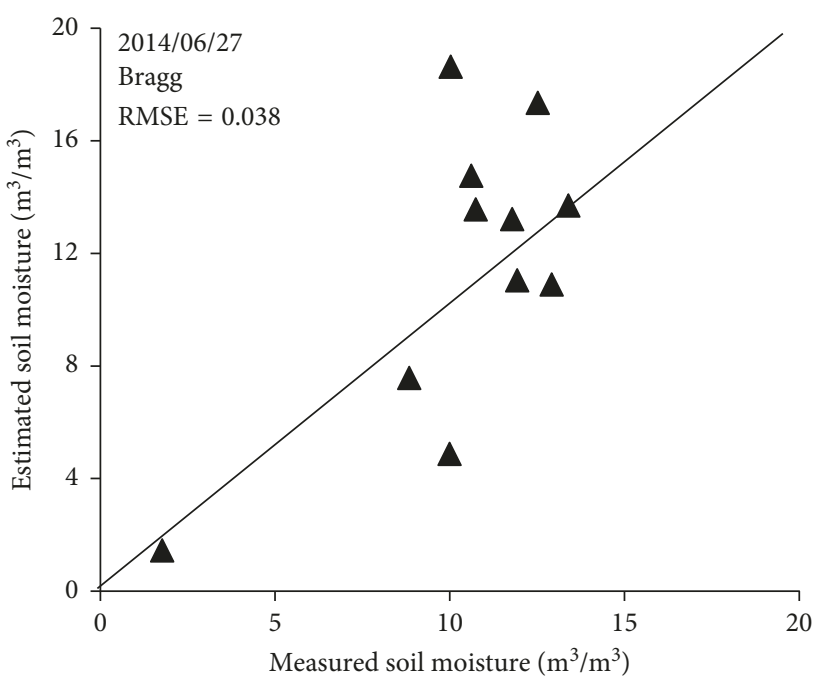

(a)

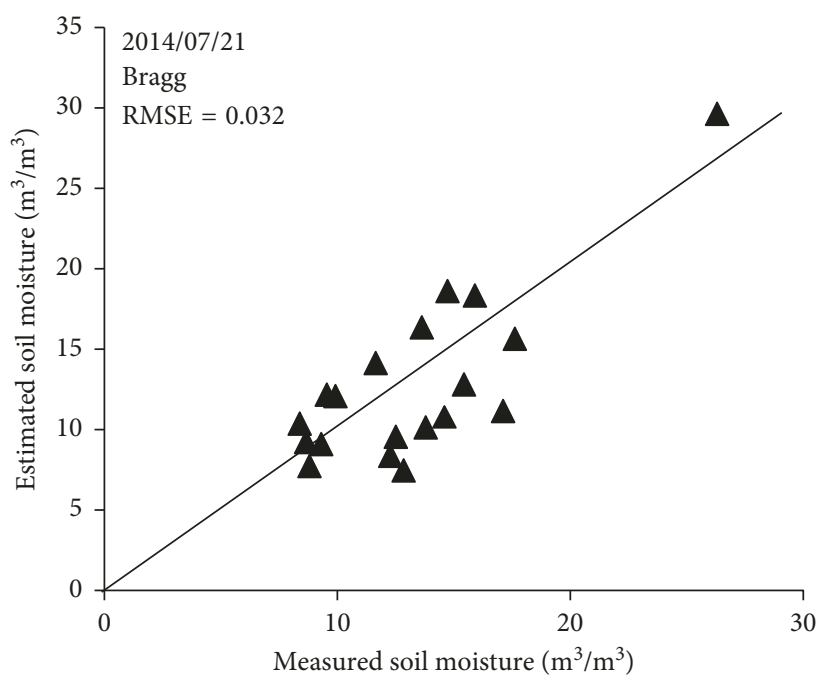

(c)

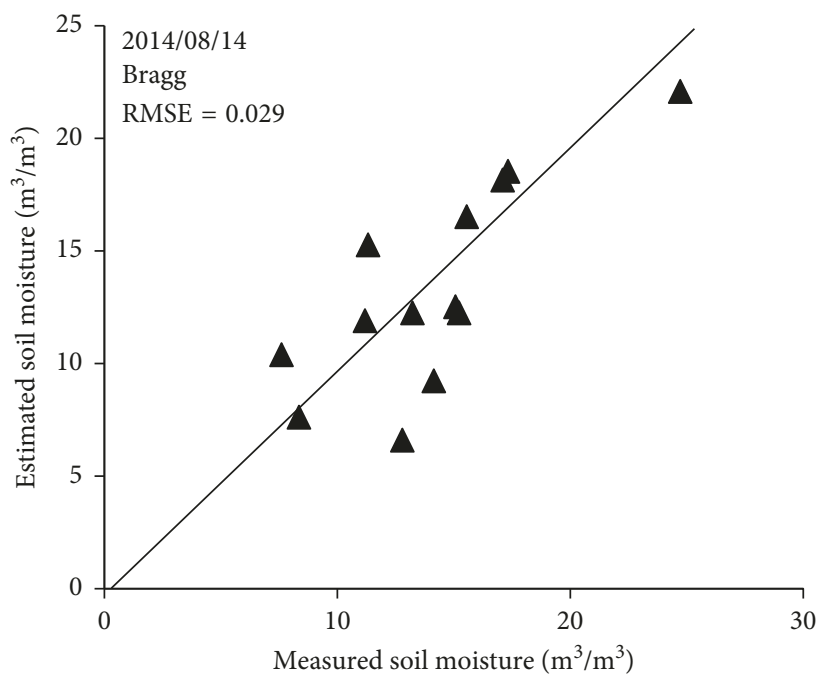

(e)

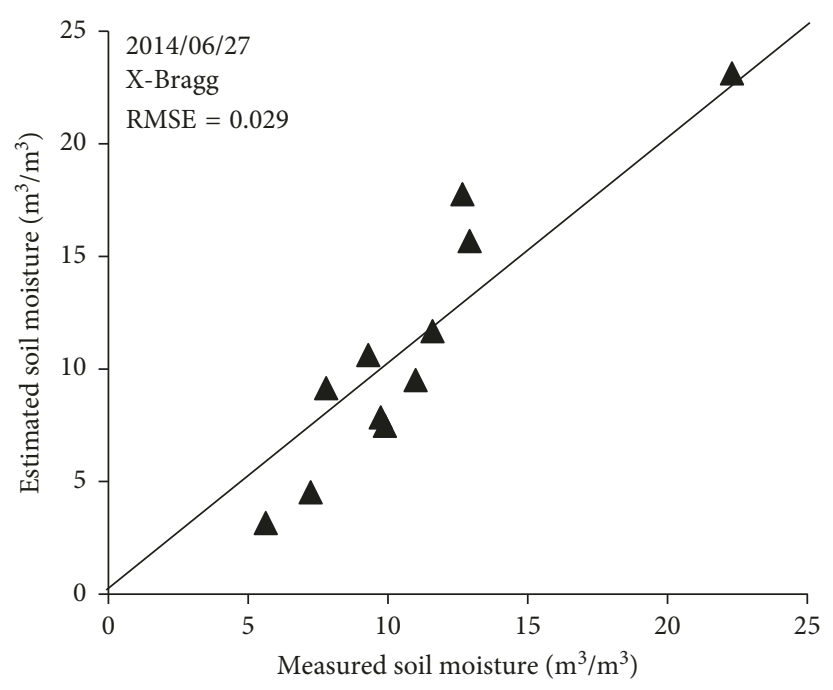

(b)

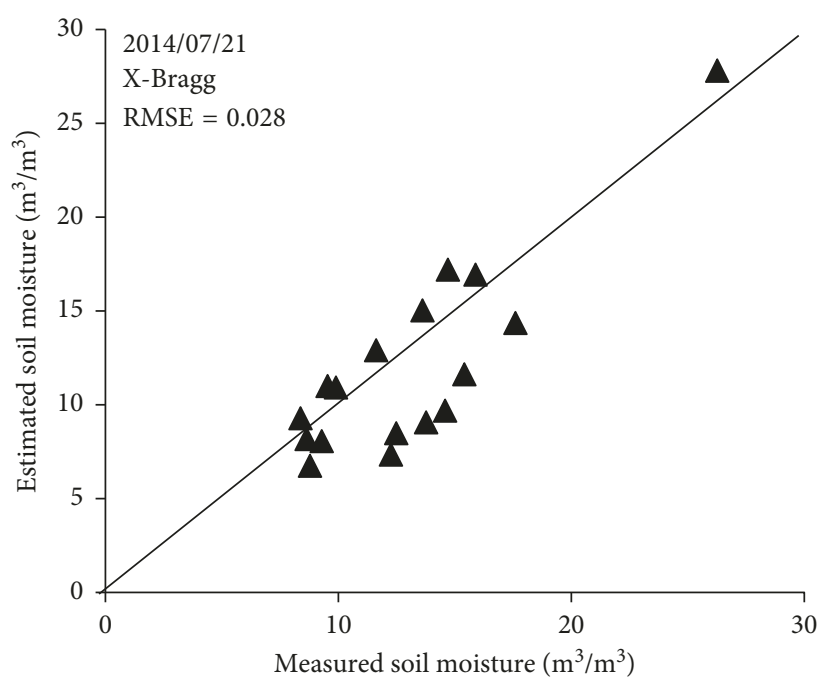

(d)

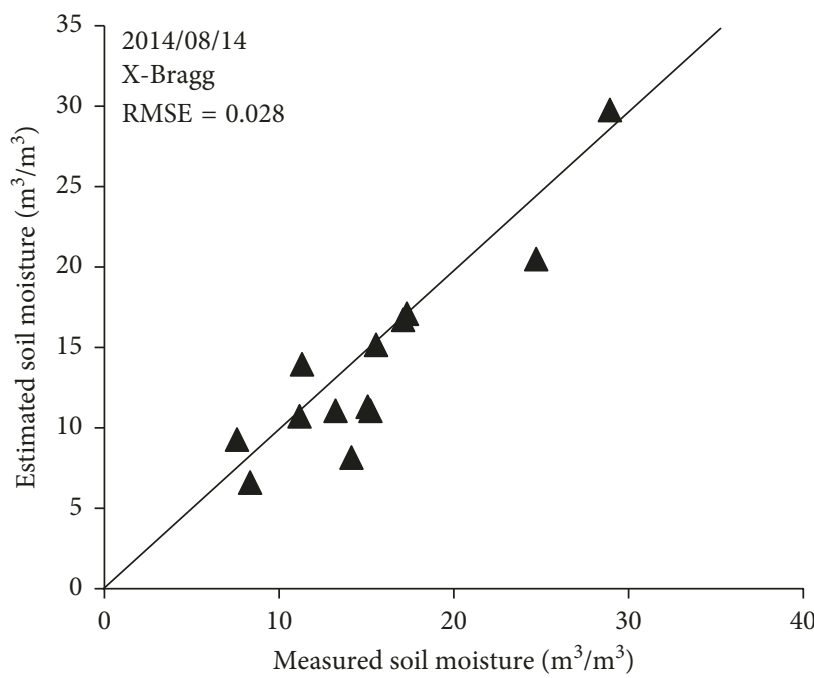

(f)

Figure 11: Continued. 


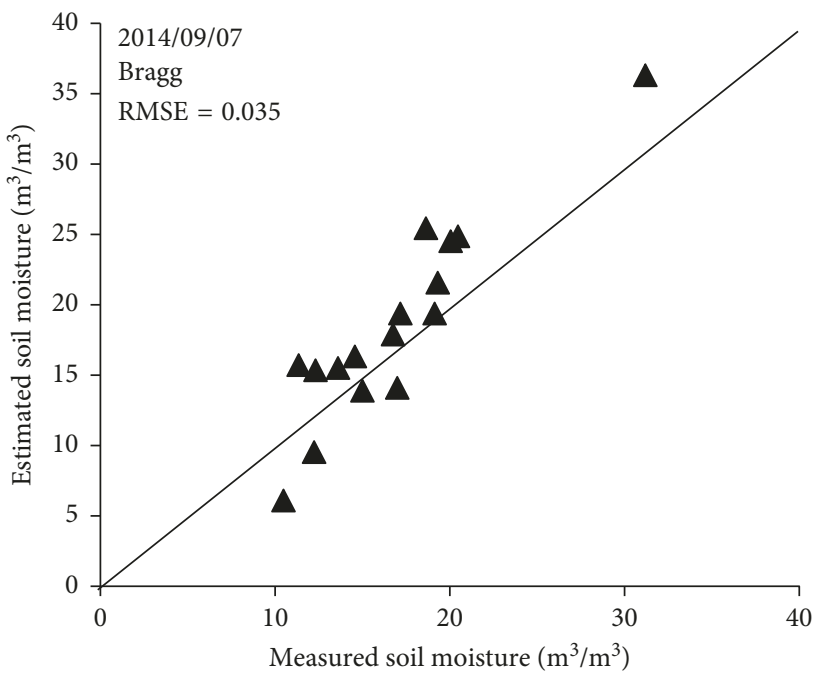

(g)

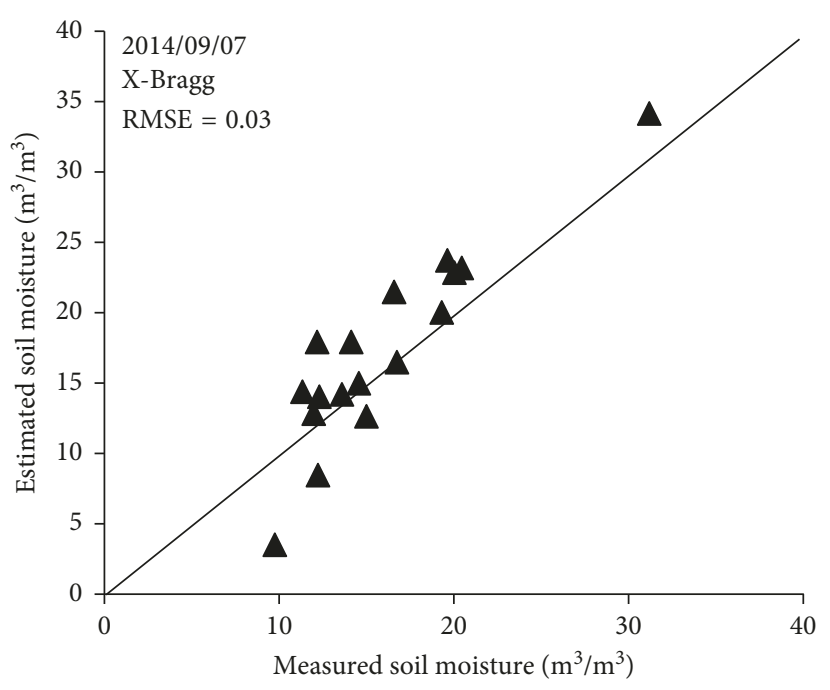

(h)

Figure 11: Accuracy of estimated soil moisture levels at different growth stages of maize.

decomposition components used in calculation (surface, dihedral, and volume scattering) are accurate. Here, we used the $H / A / A l p h a$ decomposition method to extract the surface, dihedral, and volume scattering components. The backscattering characteristics and the feature spaces of typical objects were analyzed to optimize the scattering components. We then retrieved soil moisture data using a combination of the Freeman-Durden decomposition method and the results of $H / A$ /Alpha image classification.

Our analysis shows that the backscattering characteristics of maize and cotton changed with growth in a manner expressed by our regression equations (Figures 3 and 5). Based on the threshold values of typical objects as revealed by the $H / A$ /Alpha polarization decomposition method, the nature of the surface and the volume of vegetation (if any) were apparent in the space plots of both $H$ and Alpha. Bare soil data clustered in the lower left corner of the feature space and vegetation data in the upper right corner. Thus, the $H$ and Alpha values of bare soil are lower than those of vegetation. The threshold values are approximately $0.5 \mathrm{H}$ and $25^{\circ}$ Alpha. Therefore, the thresholds of the three scattering components are $0.2-0.4 \mathrm{H}$ and $7-24^{\circ}$ Alpha for the surface scattering component; $0.6-0.9 H$ and $22-50^{\circ}$ Alpha for the volume scattering component, and other values for the dihedral scattering component. We compared the soil moisture retrieval data obtained using the Bragg and X-Bragg models, employing the FreemanDurden polarization decomposition method, in fields in which maize was at different stages of growth. The X-Bragg data of June 27, 2014, were better (having the lowest RMSE value, 0.028 ) than those derived at times when maize was at other stages of growth. The data obtained from July to September 2014 were less reliable because the C-band penetrated poorly over this time interval. However, our algorithm shows great potential for retrieval of soil moisture data using L-band polarization.

\section{Data Availability}

The data used to support the findings of this study are available from the corresponding author upon request.

\section{Conflicts of Interest}

The authors declare that they have no conflicts of interest.

\section{Authors' Contributions}

This study was performed with collaboration among all the authors. Qiuxia Xie and Qingyan Meng determined the research theme and developed the methodology of soil moisture retrieval. Linlin Zhang, Chuimei Wang, Qiao Wang, and Shaohua Zhao were involved in the field experiments and paper modification. All authors agreed to the manuscript being submitted.

\section{Acknowledgments}

The authors would like to thank the National Key Research and Development Program (China's 13th Five Year Plan) "Spatial information service and application demonstration of comprehensive monitoring of urban and rural ecological environment" (2017YFB0503900); the National Key Research and Development Program (China's 13th Five Year Plan) "Integration and application demonstration of comprehensive monitoring technology of urban and rural ecological environment" (2017YFB0503905); Hainan Province Natural Science Foundation: Urban Impervious Surface Remote Sensing Extraction and Study on the Characteristics of Multi Temporal and Spatial Evolution (417218); Hainan Province Natural Science Foundation: Urban Heat Island Remote Sensing Retrieval Technology in Urban Main Construction Area based on Night Light Index 
(417219); and National Natural Science Foundation Project (41501400).

\section{References}

[1] H. Xu, F. Zheng, and W. Zhang, "Variability in dust observed over China using A-train CALIOP instrument," Advances in Meteorology, vol. 2016, Article ID 1246590, 11 pages, 2016.

[2] H. Xu, T. Cheng, X. Gu, T. Yu, D. Xie, and F. Zheng, "Spatiotemporal variability in dust observed over the Sinkiang and Inner Mongolia regions of Northern China," Atmospheric Pollution Research, vol. 6, no. 4, pp. 562-571, 2015.

[3] H. Xu, T. Cheng, X. Gu, T. Yu, Y. Wu, and H. Chen, "New Asia dust storm detection method based on the thermal infrared spectral signature," Remote Sensing, vol. 7, no. 1, pp. 51-71, 2014.

[4] S. Tomer, A. Al Bitar, M. Sekhar et al., "Retrieval and multiscale validation of soil moisture from multi-temporal SAR data in a semi-arid tropical region," Remote Sensing, vol. 7, no. 6, pp. 8128-8153, 2015.

[5] E. P. W. Attema and F. T. Ulaby, "Vegetation modeled as a water cloud," Radio Science, vol. 13, no. 2, pp. 357-364, 1978.

[6] N. N. Baghdadi, M. El Hajj, M. Zribi, and I. Fayad, "Coupling SAR C-band and optical data for soil moisture and leaf area index retrieval over irrigated grasslands," IEEE Journal of Selected Topics in Applied Earth Observations and Remote Sensing, vol. 9, no. 3, pp. 1229-1243, 2016.

[7] G. Bertoldi, S. Della Chiesa, C. Notarnicola, L. Pasolli, G. Niedrist, and U. Tappeiner, "Estimation of soil moisture patterns in mountain grasslands by means of SAR RADARSAT2 images and hydrological modeling," Journal of Hydrology, vol. 516, pp. 245-257, 2014.

[8] I. Gherboudj, R. Magagi, A. A. Berg, and B. Toth, "Soil moisture retrieval over agricultural fields from multipolarized and multi-angular RADARSAT-2 SAR data," Remote Sensing of Environment, vol. 115, no. 1, pp. 33-43, 2011.

[9] T. Jagdhuber, I. Hajnsek, and K. P. Papathanassiou, "An iterative generalized hybrid decomposition for soil moisture retrieval under vegetation cover using fully polarimetric SAR," IEEE Journal of Selected Topics in Applied Earth Observations and Remote Sensing, vol. 8, no. 8, pp. 3911-3922, 2015.

[10] Y. Kumar, S. Singh, R. S. Chatterjee, and M. Trivedi, "A comparative analysis of extended water cloud model and backscatter modelling for above-ground biomass assessment in Corbett Tiger Reserve," in Proceedings of the SPIE AsiaPacific Remote Sensing, vol. 9880, New Delhi, India, April 2016.

[11] J. X. Zhang, G. M. Huang, J. J. Wei, and Z. Zhao, "Alternative to four-component decomposition for polarimetric SAR," ISPRS Annals of Photogrammetry, Remote Sensing and Spatial Information Sciences, vol. III-7, pp. 207-211, 2016.

[12] A. Freeman and S. L. Durden, "A three-component scattering model for polarimetric SAR data," IEEE Transactions on Geoscience and Remote Sensing, vol. 36, no. 3, pp. 963-973, 1996.

[13] A. Freeman and S. L. Durden, "Three-component scattering model to describe polarimetric SAR data," in Proceedings of the SPIE, Radar Polarimetry, vol. 1748, pp. 213-224, San Diego, CA, USA, February 1993.
[14] C. Fang and H. Wen, "A new classification method based on Cloude Pottier eigenvalue/eigenvector decomposition," in Proceedings of the IEEE International Geoscience and Remote Sensing Symposium, IGARSS'05, p. 4, Seoul, Korea, July 2005.

[15] R. Cloude and K. P. Papathanassiou, "Surface roughness and polarimetric entropy," in Proceedings of the International Geoscience and Remote Sensing Symposium, IGARSS'99, vol. 5, pp. 2443-2445, Hamburg, Germany, June-July 1999.

[16] F. Cao, W. Hong, and Y. R. Wu, "Unsupervised classification for fully polarimetric SAR data using Cloude-Pottier decomposition and agglomerative hierarchical clustering algorithm," Acta Electronica Sinica, vol. 36, no. 3, pp. 543-546, 2008.

[17] Q. Xie, Q. Meng, L. Zhang, C. Wang, Y. Sun, and Z. Sun, “A soil moisture retrieval method based on typical polarization decomposition techniques for a maize field from fullpolarization Radarsat-2 data," Remote Sensing, vol. 9, no. 2, p. 168, 2017.

[18] L. Pasolli, C. Notarnicola, L. Bruzzone et al., "Polarimetric RADARSAT-2 imagery for soil moisture retrieval in alpine areas," Canadian Journal of Remote Sensing, vol. 37, no. 5, pp. 535-547, 2011.

[19] H. Lievens and N. E. C. Verhoest, "Spatial and temporal soil moisture estimation from RADARSAT-2 imagery over Flevoland, The Netherlands," Journal of Hydrology, vol. 456-457, pp. 44-56, 2012.

[20] Q. B. Zhou, Y. U. Qiang-Yi, L. Jia, W. U. Wen-Bin, and H. J. Tang, "Perspective of Chinese GF-1 high-resolution satellite data in agricultural remote sensing monitoring," Journal of Integrative Agriculture, vol. 16, no. 2, pp. 242-251, 2017.

[21] X. Zhang and N. Chen, "Reconstruction of GF-1 soil moisture observation based on satellite and in situ sensor collaboration under full cloud contamination," IEEE Transactions on Geoscience and Remote Sensing, vol. 54, no. 9, pp. 5185-5202, 2016.

[22] N. Chen, J. Li, and X. Zhang, "Quantitative evaluation of observation capability of GF-1 wide field of view sensors for soil moisture inversion," Journal of Applied Remote Sensing, vol. 9, no. 1, article 097097, 2015.

[23] Y. Guo, W. U. Xi-Hong, Y. Z. Cheng, L. G. Wang, and T. Liu, "Maize recognition and accuracy evaluation based on high resolution remote sensing (GF-1) data," Remote Sensing Information, vol. 30, no. 6, pp. 31-36, 2015.

[24] P. Wang, Z. F. Zhou, J. Liao, and G. N. University, "Study on soil moisture retrieval of tobacco field in Karst plateau mountainous area based on freeman decomposition," Geography and Geo-Information Science, vol. 32, pp. 72-76, 2016.

[25] I. Brown, S. Mwansasu, and L. Westerberg, "L-band polarimetric target decomposition of mangroves of the Rufiji Delta, Tanzania," Remote Sensing, vol. 8, no. 2, p. 140, 2016.

[26] H. Kankwamba, J. Mangisoni, F. Simtowe, and K. Mausch, "Quantitative estimation of surface soil moisture in agricultural landscapes using spaceborne synthetic aperture radar imaging at different frequencies and polarizations," Ph.D. thesis, Natural Sciences and Mathematics, Universität zu Köln, Cologne, Germany, 2011.

[27] N. Baghdadi, R. Cresson, E. Pottier et al., "A potential use for the c-band polarimetric SAR parameters to characterize the soil surface over bare agriculture fields," IEEE Transactions On Geoscience and Remote Sensing, vol. 50, no. 10, pp. 3844-3858, 2012. 
[28] S. R. Cloude, "Polarimetry: the characterisation of polarisation effects in EM scattering," Ph.D. thesis, University of Birmingham, Birmingham, UK, 1986.

[29] N. Baghdadi, P. Dubois-Fernandez, X. Dupuis, and M. Zribi, "Sensitivity of main polarimetric parameters of multifrequency polarimetric SAR data to soil moisture and surface roughness over bare agricultural soils," IEEE Geoscience and Remote Sensing Letters, vol. 10, no. 4, pp. 731-735, 2013.

[30] T. Jagdhuber, I. Hajnsek, A. Bronstert, and K. P. Papathanassiou, "Soil moisture estimation under low vegetation cover using a multi-angular polarimetric decomposition," IEEE Transactions On Geoscience and Remote Sensing, vol. 51, no. 4, pp. 2201-2215, 2013.

[31] A. Freeman, S. Durden, and R. Zimmerman, "Mapping subtropical vegetation using multi-frequency, multi-polarization SAR data," in Proceedings of the International Geoscience and Remote Sensing Symposium, IGARSS'92, pp. 1686-1689, Houston, TX, USA, May 1992.

[32] H. Wang, R. Magagi, and K. Goita, "Comparison of different polarimetric decompositions for soil moisture retrieval over vegetation covered agricultural area," Remote Sensing of Environment, vol. 199, pp. 120-136, 2017.

[33] H. Wang, R. Magagi, K. Goita, T. Jagdhuber, and I. Hajnsek, "Evaluation of simplified polarimetric decomposition for soil moisture retrieval over vegetated agricultural fields," Remote Sensing, vol. 8, no. 2, p. 142, 2016.

[34] H. Wang, R. Magagi, K. Goita, T. Jagdhuber, and N. Djamai, "Evaluation of polarimetric decomposition for soil moisture retrieval over vegetation agriculture fields," in Proceedings of the IEEE International Geoscience and Remote Sensing Symposium, IGARSS'15, pp. 689-692, Milan, Italy, July 2015.

[35] X. U. Xing-Ou and N. Shu, "Water content information extraction from quad-polarization SAR images via Freeman-Durden decomposition," Computer Engineering and Applications, vol. 46, no. 27, pp. 17-20, 2010.

[36] J. C. Shi, J. S. Lee, K. Chen, and Q. Q. Sun, "Evaluate usage of decomposition technique in estimation of soil moisture with vegetated surface by multi-temporal measurements," in Proceedings of the IEEE 2000 International Geoscience and Remote Sensing Symposium, IGARSS 2000, pp. 1098-1100, Honolulu, HI, USA, July 2000. 

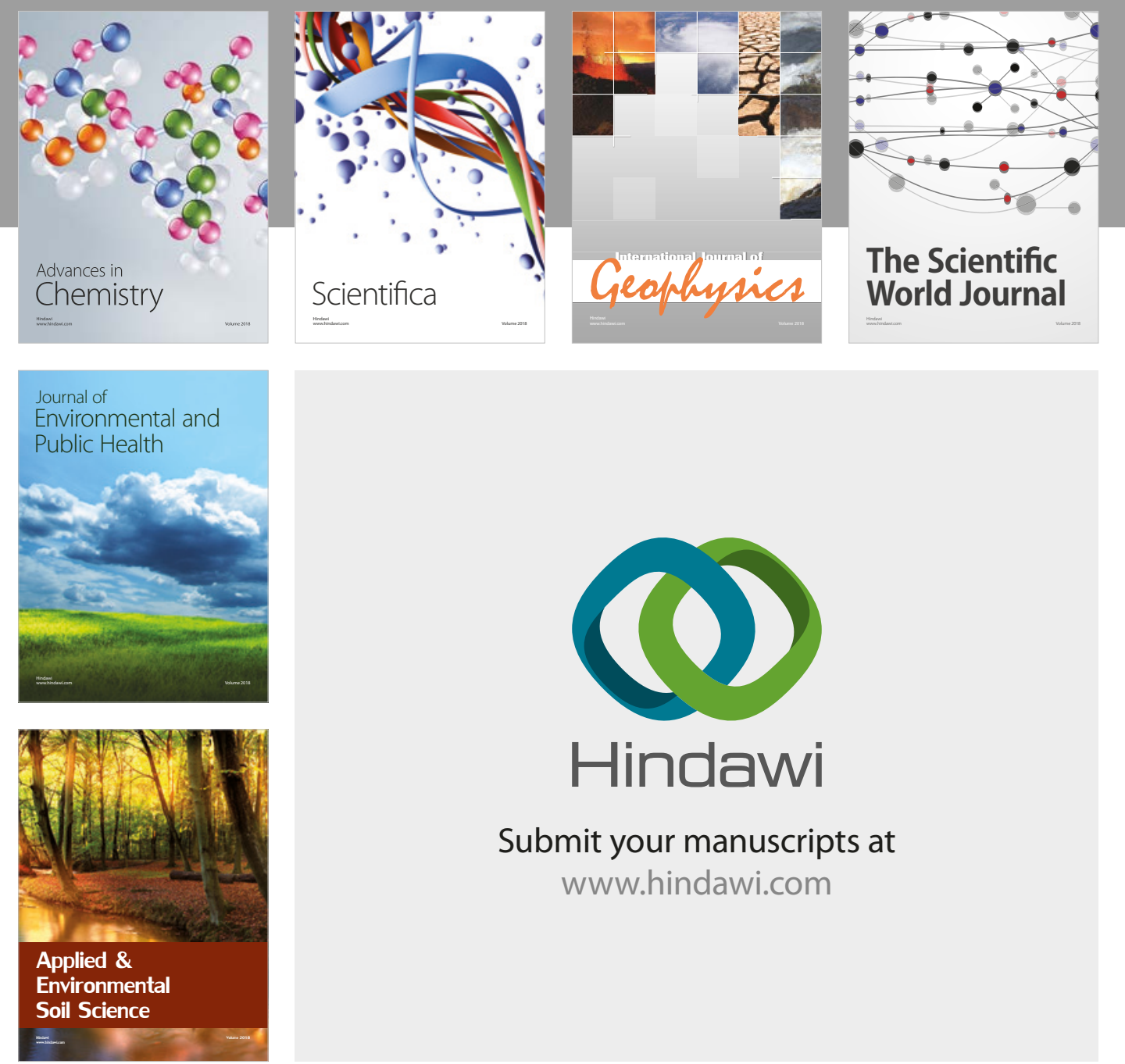

The Scientific

\section{World Journal}
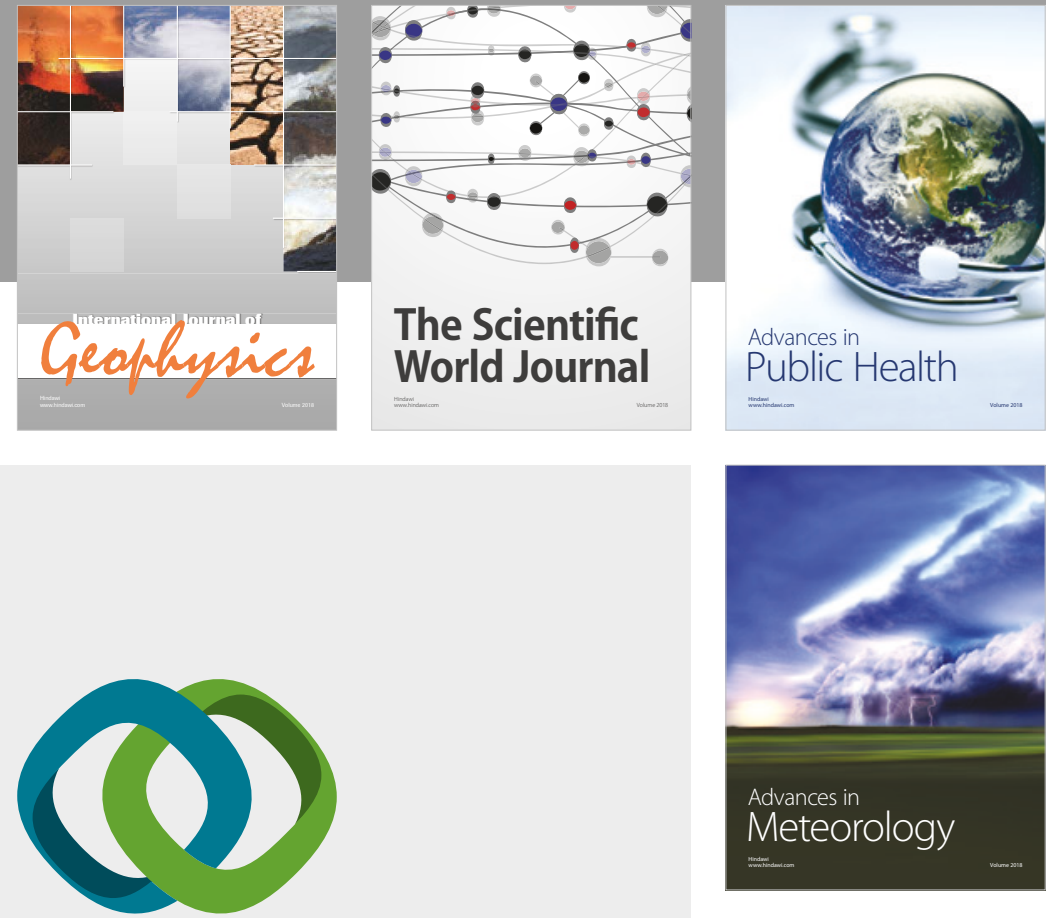

Advan

Public Health

\section{Hindawi}

Submit your manuscripts at

www.hindawi.com
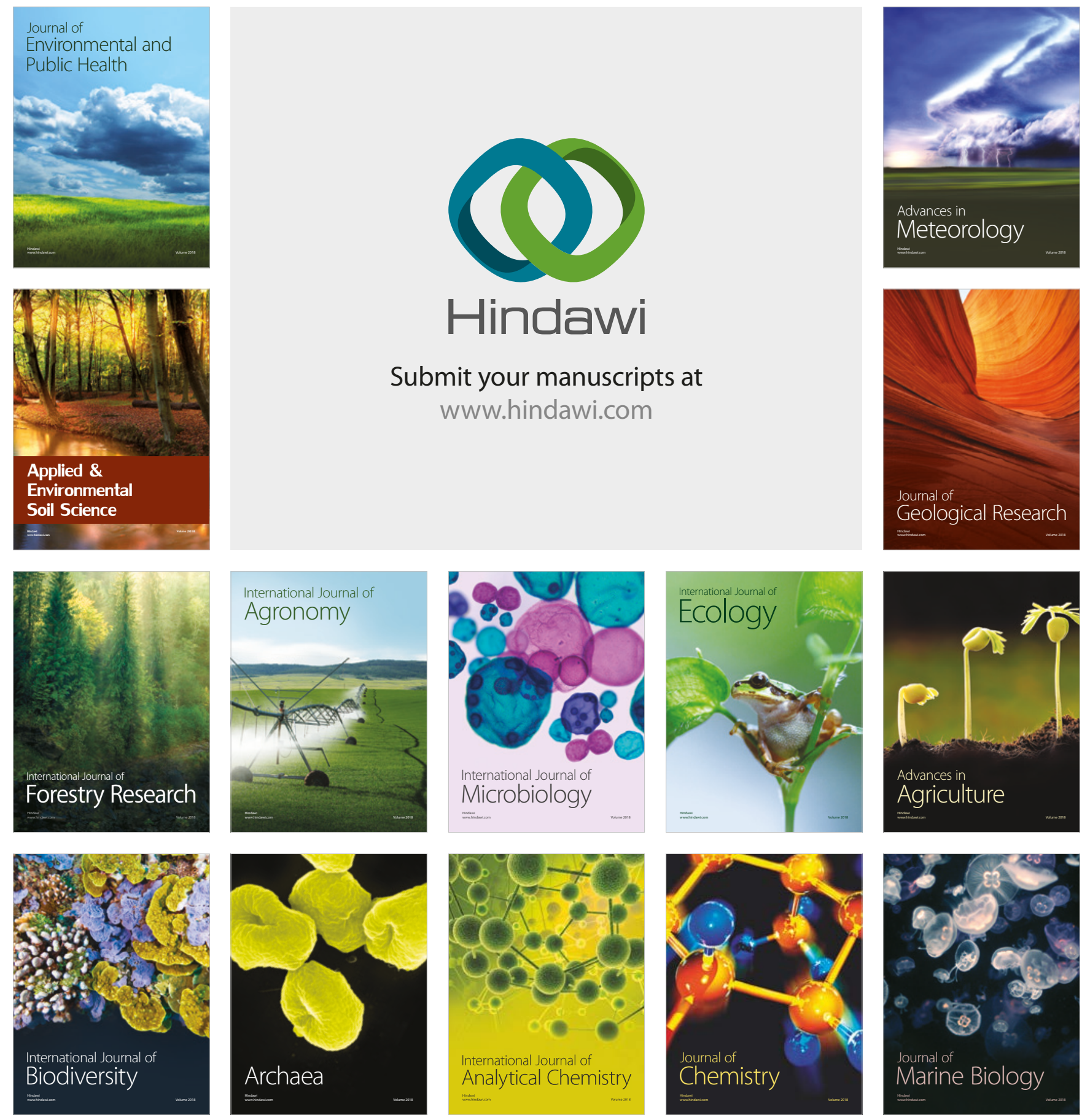